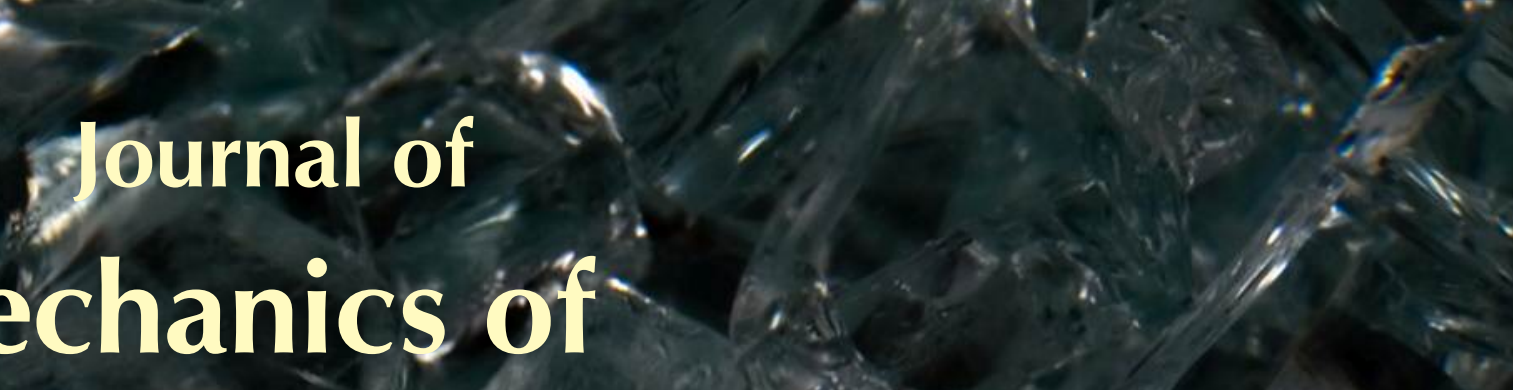

Materials and Structures

Mechanics of :

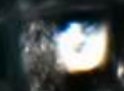

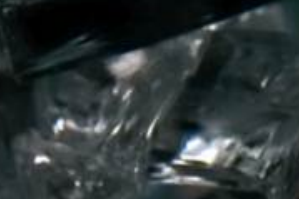

$+x^{\circ}$
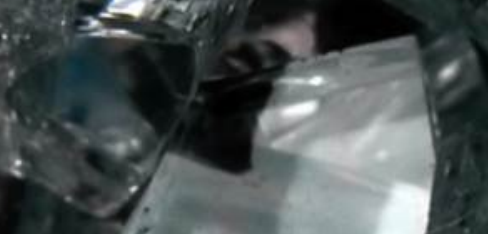

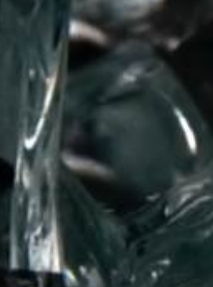
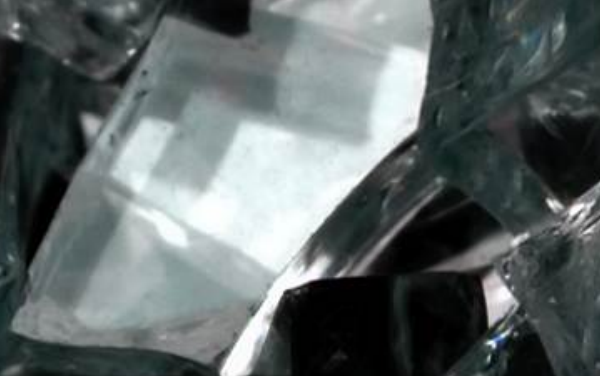

MOLECULAR DYNAMICS-BASED CONTINUUM MODELS FONUHELINEAR ELASTICITY OF NANOFILMS AND NANOWRRS WITH ANISOTROPIC ? 1. II SURFACE EFFECTS

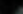

Wonbae Kim, Seung Yun Rhee and Maenghyo Cho

,

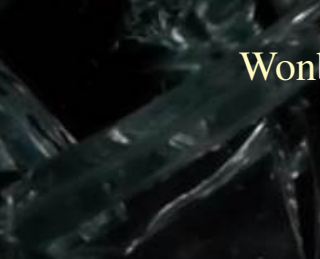

2

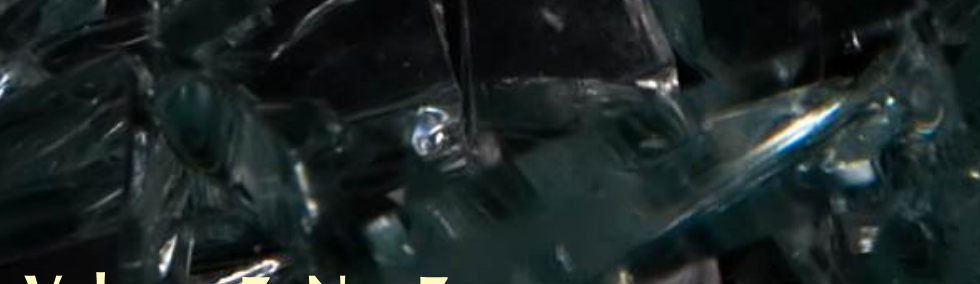

Volume 7, No. 7 ,
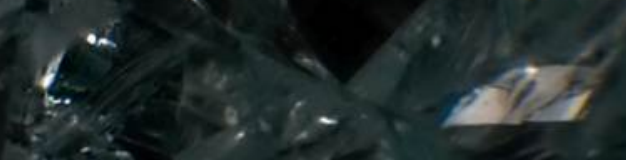


\title{
MOLECULAR DYNAMICS-BASED CONTINUUM MODELS FOR THE LINEAR ELASTICITY OF NANOFILMS AND NANOWIRES WITH ANISOTROPIC SURFACE EFFECTS
}

\author{
Wonbae Kim, Seung Yun Rhee And Maenghyo Cho
}

\begin{abstract}
A continuum-based sequential multiscale model is presented for application to nanofilms and nanowires with anisotropic surfaces. The surface effect is accounted for via the inclusion of surface energy due to surface stress and surface strain in the internal energy. For anisotropic surfaces such as a $<110>$ surface, a linear surface elasticity model is used instead of the isotropic surface elasticity model proposed by Gurtin and Murdoch. A molecular dynamics simulation is performed in order to calculate initial surface stress and surface elastic tensor. Equilibrium strain and size-dependent elasticity are estimated by the proposed continuum model.
\end{abstract}

\section{Introduction}

As more and more nanoscaled structures such as nanofilms and nanowires are fabricated, it is becoming more important to understand the mechanical characteristics of nanostructures. It is well known that the mechanical properties of a nanosized structure significantly differ from those of bulk materials because of the surface effect. Therefore, although the surface effect is negligible in macroscale studies, it is crucial for a nanosized structure analysis to account for the influence of the surface effect [Miller and Shenoy 2000; Zhou and Huang 2004; Diao et al. 2004; Liang et al. 2005; Guo and Zhao 2005; Sun and Zhang 2005; Dingreville et al. 2005; Wang et al. 2008; He and Lilley 2008; Zhang et al. 2008; Dingreville et al. 2008; Park 2009; Kim and Cho 2010].

An atom on a free surface of a crystal structure has a different coordination number (i.e. the number of nearest neighbors) from an atom in the bulk material. For this reason, solid crystal surfaces contain excess surface energy and residual surface stress. The surface stress significantly affects the equilibrium state and elastic properties of a nanostructure [Wolf 1991; Cammarata and Sieradzki 1989; Streitz et al. 1994]. Streitz et al. [1994] introduced a surface stress model and calculated the equilibrium biaxial strain and biaxial modulus for an axis-symmetric thin film. Miller and Shenoy [2000] estimated the effective stiffnesses of nanosized structures using atomistic calculations. Recently, Dingreville et al. [2008] proposed a semi-analytical method for quantifying the size-dependent elasticity of nanostructures, and Kim and Cho [2010] computed equilibrium strain and size-dependent elasticity using a surface relaxation model and a new surface stress model.

As one of the continuum approaches, Gurtin and Murdoch [1975; 1978] introduced a surface elasticity model for isotropic surfaces using initial surface stress and surface Lamé constants. A plate theory based on Kirchhoff and Mindlin plate theories, which included the surface effect by using Gurtin and

Keywords: surface effects, sequential multiscale model, finite element method, nanofilm, nanowire, size-dependent elasticity, molecular dynamics simulation. 
Murdoch's surface model, was proposed by Lim and He [2004] and Lu et al. [2006]. Recently, Cho et al. [2009] proposed a continuum-based bridging model for a nanofilm using the surface elasticity model by Gurtin and Murdoch [1975; 1978]. Cho et al. [2009] suggested a sequential multiscale method in order to determine initial surface stress and surface Lamé constants using a matching method based on a molecular dynamics (MD) simulation. As an extension of this multiscale model, a finite element analysis including thermomechanical properties was carried out by Choi et al. [2010a].

With the exception of $\{111\}$ surfaces, FCC crystal surfaces are not isotropic; for example, a $\{100\}$ surface and a $\{110\}$ surface of an FCC single crystal are orthotropic. Therefore, the surface elasticity model developed by Gurtin and Murdoch cannot be universally applied to single crystal surfaces. Choi et al. [2010b] modified Gurtin and Murdoch's model, adding an additional parameter to two surface Lamé constants for the analysis of a nanofilm resonator with a $\{100\}$ surface. However, this modified surface elasticity is not applicable to a $\{110\}$ surface. Although there is need for a multiscale continuum model that can be applied to general nanostructures, no single model can handle all anisotropic surfaces.

This paper presents a new continuum-based sequential multiscale model for nanofilms and nanowires with anisotropic surfaces. This model utilizes a linear surface elastic model, which is represented in terms of initial surface stress and surface elastic tensor, instead of relying on Gurtin and Murdoch's surface model. The surface parameters, initial surface stress and surface elastic tensor, are determined by applying a matching technique to the results from an MD simulation.

\section{Multiscale continuum model including surface effect}

2.1. Multiscale continuum model for a nanofilm. Mindlin plate theory is adopted for the proposed model. For a nanofilm with top $\left(\Omega^{+}\right)$and bottom $\left(\Omega^{-}\right)$surfaces, as shown in Figure 1, the displacement field based on Mindlin plate theory is defined as

$$
\begin{aligned}
& u_{\alpha}=u_{\alpha}^{0}+x_{3} \psi_{\alpha}, \\
& u_{3}=u_{3}^{0}=w,
\end{aligned}
$$

where $u_{\alpha}^{0}$ and $u_{3}^{0}$ denote in-plane and out-of-plane displacements defined in the mid-plane of the thin film, respectively, $\psi_{\alpha}$ is the rotational degree of freedom, and the subscript $\alpha$ represents in-plane directions 1

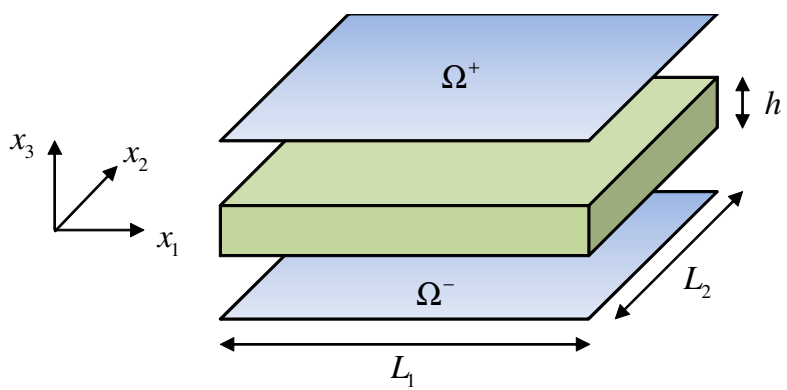

Figure 1. Schematic diagram of a nanofilm with top and bottom surfaces. 
and 2. Using this displacement field, the strain-displacement relationship, $\epsilon_{i j}=\left(u_{i, j}+u_{j, i}\right) / 2$, yields

$$
\begin{aligned}
\epsilon_{\alpha \beta} & =\frac{1}{2}\left[u_{\alpha, \beta}^{0}+u_{\beta, \alpha}^{0}+x_{3}\left(\psi_{\alpha, \beta}+\psi_{\beta, \alpha}\right)\right], \\
\epsilon_{3 \alpha} & =\frac{1}{2}\left[w_{, \alpha}+\psi_{\alpha}\right],
\end{aligned}
$$

where $u_{\alpha, 3}^{0}=0$ because $u_{\alpha}^{0}=u_{\alpha}^{0}\left(x_{1}, x_{2}\right)$. The in-plane components of the strain tensor are

$$
\begin{aligned}
\epsilon_{11} & =u_{1,1}^{0}+x_{3} \psi_{1,1}, \\
\epsilon_{22} & =u_{2,2}^{0}+x_{3} \psi_{2,2}, \\
2 \epsilon_{12} & =\left(u_{1,2}^{0}+u_{2,1}^{0}\right)+x_{3}\left(\psi_{1,2}+\psi_{2,1}\right),
\end{aligned}
$$

and the transverse shear components of the strain tensor are

$$
\begin{aligned}
& 2 \epsilon_{31}=w_{, 1}+\psi_{1}, \\
& 2 \epsilon_{32}=w_{, 2}+\psi_{2} .
\end{aligned}
$$

The displacements on the upper and lower surfaces are described as

$$
\begin{aligned}
& u_{\alpha}^{s \pm}=u_{\alpha}^{0} \pm \frac{h}{2} \psi_{\alpha}, \\
& u_{3}^{s \pm}=w,
\end{aligned}
$$

where $s+$ and $s-$ denote the upper and lower surfaces, respectively, and $h$ is the film thickness. The strain field on a surface can be written as

$$
\begin{aligned}
\epsilon_{11}^{s \pm} & =u_{1,1}^{0} \pm \frac{h}{2} \psi_{1,1}, \\
\epsilon_{22}^{s \pm} & =u_{2,2}^{0} \pm \frac{h}{2} \psi_{2,2}, \\
2 \epsilon_{12}^{s \pm} & =\left(u_{1,2}^{0}+u_{2,1}^{0}\right) \pm \frac{h}{2}\left(\psi_{1,2}+\psi_{2,1}\right) .
\end{aligned}
$$

The virtual work principle states that the internal virtual work is equal to the external virtual work, i.e. $\delta U=\delta W_{E}$. The external virtual work $\delta W_{E}$ can be written as

$$
\delta W_{E}=\int_{\Omega} p_{\alpha} \delta u_{\alpha}^{0}+p_{3} \delta w d A
$$

where $p_{\alpha}$ and $p_{3}$ are the in-plane and out-of-plane force per unit area, respectively. The internal virtual work consists of bulk energy and surface energy ( $\delta U=\delta U_{\text {bulk }}+\delta U_{\text {surf }}$ ). The bulk energy can be written in terms of displacements and resultant forces, as follows:

$$
\begin{aligned}
\delta U_{\text {bulk }} & =\int_{v} \sigma_{\alpha \beta} \delta \epsilon_{\alpha \beta} d v+\int_{v} \sigma_{3 \alpha} \delta \gamma_{3 \alpha} d v \\
& =\int_{\Omega} N_{\alpha \beta} \delta u_{\alpha, \beta}^{0}+M_{\alpha \beta} \delta \psi_{\alpha, \beta} d A+\int_{\Omega} Q_{\alpha}\left(\delta w_{, \alpha}+\delta \psi_{\alpha}\right) d A,
\end{aligned}
$$

where $\sigma_{\alpha \beta}$ and $\epsilon_{\alpha \beta}$ are in-plane stress and strain components, respectively; $\sigma_{3 \alpha}$ and $\gamma_{3 \alpha}$ are transverse shear stress and strain components, respectively; and $N_{\alpha \beta}, M_{\alpha \beta}$, and $Q_{\alpha}$ are force stress resultants, 
moment resultants, and shear stress resultants, respectively. These last three variables are defined by

$$
N_{\alpha \beta}=\int_{-h / 2}^{h / 2} \sigma_{\alpha \beta} d x_{3}, \quad M_{\alpha \beta}=\int_{-h / 2}^{h / 2} \sigma_{\alpha \beta} x_{3} d x_{3}, \quad Q_{\alpha}=\int_{-h / 2}^{h / 2} \sigma_{3 \alpha} d x_{3} .
$$

The surface energy due to surface stress is composed of both top and bottom surface energies:

$$
\delta U_{\text {surf }}=\int_{\Omega^{ \pm}} \tau_{\alpha \beta}^{ \pm} \delta \epsilon_{\alpha \beta}^{s \pm} d A=\int_{\Omega^{ \pm}} \tau_{\alpha \beta}^{ \pm} \delta\left(u_{\alpha, \beta}^{0} \pm \frac{h}{2} \psi_{\alpha, \beta}\right) d A,
$$

where $\Omega^{+}$and $\Omega^{-}$represent the top and bottom surfaces, respectively; $\tau_{\alpha \beta}^{s \pm}$ is a surface stress component; and $\epsilon_{\alpha \beta}^{s \pm}$ is a surface strain component. Using (8) and (10), the internal virtual work yields

$$
\begin{aligned}
\delta U & =\delta U_{\text {bulk }}+\delta U_{\text {surf }} \\
& =\int_{\Omega}\left(N_{\alpha \beta}+\tau_{\alpha \beta}^{+}+\tau_{\alpha \beta}^{-}\right) \delta u_{\alpha, \beta}^{0} d A+\int_{\Omega}\left(M_{\alpha \beta}+\frac{h}{2}\left(\tau_{\alpha \beta}^{+}-\tau_{\alpha \beta}^{-}\right)\right) \delta \psi_{\alpha, \beta} d A+\int_{\Omega} Q_{\alpha}\left(\delta w_{, \alpha}+\delta \psi_{\alpha}\right) d A .
\end{aligned}
$$

The linear elastic constitutive equation, $\sigma_{i j}=C_{i j k l} \epsilon_{k l}$, is utilized for the bulk material, and the following relationship is used to determine the surface constitutive relationship:

$$
\tau_{\alpha \beta}^{ \pm}=\tau_{\alpha \beta}^{0}+T_{\alpha \beta \kappa \lambda} \epsilon_{\kappa \lambda}^{s \pm},
$$

where $\tau_{\alpha \beta}^{0}$ is the initial surface stress component at zero strain and $T_{\alpha \beta \kappa \lambda}$ is the surface elastic tensor component. This linear surface constitutive equation can be used for any anisotropic surface, whereas Gurtin and Murdoch's surface elasticity can be applied only to isotropic surfaces.

Finite element implementation for a nanofilm. When implementing a finite element analysis for a nanofilm, an isoparametric 4-node element is used. The nodal displacement is defined as

$$
\boldsymbol{d}=\left[\left(u_{1}, u_{2}, w, \psi_{1}, \psi_{2}\right)_{1}, \ldots,\left(u_{1}, u_{2}, w, \psi_{1}, \psi_{2}\right)_{n}\right]^{T},
$$

and the displacement field is interpolated within nodal values.

$$
u_{\alpha}^{0}=\sum_{i=1}^{n} N_{i}\left(u_{\alpha}\right)_{i}, \quad w=\sum_{i=1}^{n} N_{i} w_{i}, \quad \psi_{\alpha}=\sum_{i=1}^{n} N_{i}\left(\psi_{\alpha}\right)_{i},
$$

where $N_{i}$ is the $i$-th interpolation function and $n$ is the number of nodes per element. Using these nodal displacements, the strain field can be rewritten as

$$
\begin{gathered}
\left\{\begin{array}{c}
\epsilon_{11} \\
\epsilon_{22} \\
2 \epsilon_{12}
\end{array}\right\}=\left\{\begin{array}{c}
u_{1,1}^{0} \\
u_{2,2}^{0} \\
u_{1,2}^{0}+u_{2,1}^{0}
\end{array}\right\}+x_{3}\left\{\begin{array}{c}
\psi_{1,1} \\
\psi_{2,2} \\
\psi_{1,2}+\psi_{2,1}
\end{array}\right\}=\boldsymbol{B}_{m} \boldsymbol{d}+x_{3} \boldsymbol{B}_{b} \boldsymbol{d}, \\
\left\{\begin{array}{l}
2 \epsilon_{31} \\
2 \epsilon_{32}
\end{array}\right\}=\left\{\begin{array}{l}
w_{, 1}+\psi_{1} \\
w_{, 2}+\psi_{2}
\end{array}\right\}=\boldsymbol{B}_{s} \boldsymbol{d},
\end{gathered}
$$


where matrices $\boldsymbol{B}_{m}, \boldsymbol{B}_{b}$, and $\boldsymbol{B}_{s}$ represent the relationships between nodal displacement and membrane, bending, and transverse shear strains, as follows:

$$
\begin{aligned}
& \boldsymbol{B}_{m} \boldsymbol{d}=\left[\boldsymbol{B}_{m}^{(1)}, \boldsymbol{B}_{m}^{(2)}, \ldots, \boldsymbol{B}_{m}^{(n)}\right] \boldsymbol{d}=\left\{\begin{array}{c}
u_{1,1}^{0} \\
u_{2,2}^{0} \\
u_{1,2}^{0}+u_{2,1}^{0}
\end{array}\right\}, \\
& \boldsymbol{B}_{b} \boldsymbol{d}=\left[\boldsymbol{B}_{b}^{(1)}, \boldsymbol{B}_{b}^{(2)}, \ldots, \boldsymbol{B}_{b}^{(n)}\right] \boldsymbol{d}=\left\{\begin{array}{c}
\psi_{1,1} \\
\psi_{2,2} \\
\psi_{1,2}+\psi_{2,1}
\end{array}\right\}, \\
& \boldsymbol{B}_{s} \boldsymbol{d}=\left[\boldsymbol{B}_{s}^{(1)}, \boldsymbol{B}_{s}^{(2)}, \ldots, \boldsymbol{B}_{s}^{(n)}\right] \boldsymbol{d}=\left\{\begin{array}{c}
w_{, 1}+\psi_{1} \\
w_{, 2}+\psi_{2}
\end{array}\right\},
\end{aligned}
$$

and

$$
\boldsymbol{B}_{m}^{(i)}=\left[\begin{array}{ccccc}
N_{i, x} & 0 & 0 & 0 & 0 \\
0 & N_{i, y} & 0 & 0 & 0 \\
N_{i, y} & N_{i, x} & 0 & 0 & 0
\end{array}\right], \quad \boldsymbol{B}_{b}^{(i)}=\left[\begin{array}{ccccc}
0 & 0 & 0 & N_{i, x} & 0 \\
0 & 0 & 0 & 0 & N_{i, y} \\
0 & 0 & 0 & N_{i, y} & N_{i, x}
\end{array}\right], \quad \boldsymbol{B}_{s}^{(i)}=\left[\begin{array}{ccccc}
0 & 0 & N_{i, x} & N_{i} & 0 \\
0 & 0 & N_{i, y} & 0 & N_{i}
\end{array}\right] .
$$

The constitutive equations of in-plane and transverse shear parts are

$$
\begin{gathered}
\left\{\begin{array}{l}
\sigma_{11} \\
\sigma_{22} \\
\sigma_{12}
\end{array}\right\}=[\boldsymbol{Q}]\left\{\begin{array}{c}
\epsilon_{11} \\
\epsilon_{22} \\
2 \epsilon_{12}
\end{array}\right\}, \\
\left\{\begin{array}{l}
\sigma_{31} \\
\sigma_{32}
\end{array}\right\}=\left[\boldsymbol{C}_{G}\right]\left\{\begin{array}{l}
2 \epsilon_{31} \\
2 \epsilon_{32}
\end{array}\right\},
\end{gathered}
$$

where $[\boldsymbol{Q}$ ] is the $3 \times 3$ matrix representing the plane-stress constitutive relationship. [ $\boldsymbol{Q}$ ] is defined in terms of the submatrices of $[\boldsymbol{C}]$, namely

$$
\boldsymbol{C}_{a a}=\left[\begin{array}{lll}
C_{11} & C_{12} & C_{16} \\
C_{12} & C_{22} & C_{26} \\
C_{16} & C_{26} & C_{66}
\end{array}\right], \quad \boldsymbol{C}_{a b}=\left[\begin{array}{lll}
C_{13} & C_{14} & C_{15} \\
C_{23} & C_{24} & C_{25} \\
C_{63} & C_{64} & C_{65}
\end{array}\right], \quad \boldsymbol{C}_{b b}=\left[\begin{array}{lll}
C_{33} & C_{34} & C_{35} \\
C_{43} & C_{44} & C_{45} \\
C_{35} & C_{45} & C_{55}
\end{array}\right],
$$

via the formula

$$
[\boldsymbol{Q}]=\boldsymbol{C}_{a a}-\boldsymbol{C}_{a b} \boldsymbol{C}_{b b}^{-1} \boldsymbol{C}_{a b}^{T}
$$

The components $C_{i j}$ of the matrix $[\boldsymbol{C}]$ are the elastic constants written in Voigt form. Further, in (19), we have set

$$
\left[\boldsymbol{C}_{G}\right]=\left[\begin{array}{ll}
C_{55} & C_{45} \\
C_{45} & C_{44}
\end{array}\right]
$$


The internal virtual work due to force stress resultants is

$$
\begin{aligned}
\delta U_{\mathrm{bulk}}^{N_{\alpha \beta}} & =\int_{\Omega} N_{\alpha \beta} \delta u_{\alpha, \beta}^{0} d A \\
& =\delta \boldsymbol{d}^{T}\left[\int_{\Omega} \boldsymbol{B}_{m}^{T}\left(\int_{-h / 2}^{h / 2}[\boldsymbol{Q}]\left(\boldsymbol{B}_{m}+x_{3} \boldsymbol{B}_{b}\right) d x_{3}\right) d A\right] \boldsymbol{d} \\
& =\delta \boldsymbol{d}^{T}\left[\int_{\Omega} \boldsymbol{B}_{m}^{T}[\boldsymbol{A}] \boldsymbol{B}_{m}+\boldsymbol{B}_{m}^{T}[\boldsymbol{B}] \boldsymbol{B}_{b} d A\right] \boldsymbol{d},
\end{aligned}
$$

where

$$
[\boldsymbol{A}]=\int_{-h / 2}^{h / 2}[\boldsymbol{Q}] d x_{3}, \quad[\boldsymbol{B}]=\int_{-h / 2}^{h / 2}[\boldsymbol{Q}] x_{3} d x_{3} .
$$

Similarly, the internal virtual work terms due to moment resultants and shear stress resultants are

$$
\begin{aligned}
\delta U_{\text {bulk }}^{M_{\alpha \beta}} & =\int_{\Omega} M_{\alpha \beta} \delta \psi_{\alpha, \beta} d A \\
& =\delta \boldsymbol{d}^{T}\left[\int_{\Omega}\left(\int_{-h / 2}^{h / 2} x_{3} \boldsymbol{B}_{b}^{T}[\boldsymbol{Q}]\left(\boldsymbol{B}_{m}+x_{3} \boldsymbol{B}_{b}\right) d x_{3}\right) d A\right] \boldsymbol{d} \\
& =\delta \boldsymbol{d}^{T}\left[\int_{\Omega} \boldsymbol{B}_{b}^{T}[\boldsymbol{B}] \boldsymbol{B}_{m}+\boldsymbol{B}_{b}^{T}[\boldsymbol{D}] \boldsymbol{B}_{b} d A\right] \boldsymbol{d}, \\
\delta U_{\text {bulk }}^{Q_{\alpha}} & =\int_{\Omega} Q_{\alpha}\left(\delta w_{, \alpha}+\delta \psi_{\alpha}\right) d A \\
& =\delta \boldsymbol{d}^{T}\left[\int_{\Omega} k \boldsymbol{B}_{s}^{T}\left(\int_{-h / 2}^{h / 2}\left[\boldsymbol{C}_{G}\right] \boldsymbol{B}_{s} d x_{3}\right) d A\right] \boldsymbol{d} \\
& =\delta \boldsymbol{d}^{T}\left[\int_{\Omega} k \boldsymbol{B}_{s}^{T}[\boldsymbol{G}] \boldsymbol{B}_{s} d A\right] \boldsymbol{d},
\end{aligned}
$$

where

$$
[\boldsymbol{D}]=\int_{-h / 2}^{h / 2}[\boldsymbol{Q}] x_{3}^{2} d x_{3}, \quad[\boldsymbol{G}]=\int_{-h / 2}^{h / 2}\left[\boldsymbol{C}_{G}\right] d x_{3}
$$

and $k$ is the shear correction factor. Using (22), (24), and (25), the bulk internal energy can be written in the matrix form as

$$
\begin{aligned}
\delta U_{\mathrm{bulk}}=\delta U_{\mathrm{bulk}}^{N_{\alpha \beta}}+\delta U_{\mathrm{bulk}}^{M_{\alpha \beta}}+\delta U_{\mathrm{bulk}}^{Q_{\alpha}} & \\
=\delta \boldsymbol{d}^{T}\left[\int_{\Omega} \boldsymbol{B}_{m}^{T}[\boldsymbol{A}] \boldsymbol{B}_{m}+\boldsymbol{B}_{b}^{T}[\boldsymbol{D}] \boldsymbol{B}_{b} d A\right] \boldsymbol{d}+\delta \boldsymbol{d}^{T} & {\left[\int_{\Omega} k \boldsymbol{B}_{s}^{T}[\boldsymbol{G}] \boldsymbol{B}_{s} d A\right] \boldsymbol{d} } \\
& +\delta \boldsymbol{d}^{T}\left[\int_{\Omega} \boldsymbol{B}_{m}^{T}[\boldsymbol{B}] \boldsymbol{B}_{b}+\boldsymbol{B}_{b}^{T}[\boldsymbol{B}] \boldsymbol{B}_{m} d A\right] \boldsymbol{d} .
\end{aligned}
$$

Both top and bottom surface energies are considered when determining the internal virtual work due to surface energy. The surface stress term is divided into two components. The first component is 
determined by the initial surface stress at zero strain, and the second component by the surface elastic tensor. Thus,

$$
\delta U_{\text {surf }}=\int_{\Omega^{ \pm}} \tau_{\alpha \beta}^{0} \delta\left(u_{\alpha, \beta}^{0} \pm \frac{h}{2} \psi_{\alpha, \beta}\right) d A+\int_{\Omega^{ \pm}} T_{\alpha \beta \kappa \lambda}\left(u_{\kappa, \lambda}^{0} \pm \frac{h}{2} \psi_{\kappa, \lambda}\right) \delta\left(u_{\alpha, \beta}^{0} \pm \frac{h}{2} \psi_{\alpha, \beta}\right) d A .
$$

In terms of nodal displacement, the surface strain field is defined as

$$
\left\{\begin{array}{c}
\epsilon_{11}^{s \pm} \\
\epsilon_{22}^{s \pm} \\
2 \epsilon_{12}^{s \pm}
\end{array}\right\}=\boldsymbol{B}_{\tau \pm} \boldsymbol{d}=\left[\boldsymbol{B}_{\tau \pm}^{(1)}, \boldsymbol{B}_{\tau \pm}^{(2)}, \ldots, \boldsymbol{B}_{\tau \pm}^{(n)}\right] \boldsymbol{d},
$$

with

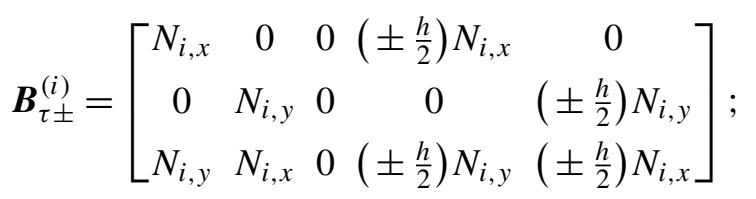

$\boldsymbol{B}_{\tau \pm}$ is the matrix that represents the relationship between surface strain and nodal displacement. The internal virtual work due to the surface energy can be written in the following discrete form:

$$
\begin{aligned}
\delta U_{\text {surf }}=\delta \boldsymbol{d}^{T} \int_{\Omega^{+}} \boldsymbol{B}_{\tau+}^{T}\left\{\tau^{0}\right\} d A+\delta \boldsymbol{d}^{T} & \int_{\Omega^{-}} \boldsymbol{B}_{\tau-}^{T}\left\{\tau^{0}\right\} d A \\
& +\delta \boldsymbol{d}^{T}\left[\int_{\Omega^{+}} \boldsymbol{B}_{\tau+}^{T}[\boldsymbol{T}] \boldsymbol{B}_{\tau+} d A\right] \boldsymbol{d}+\delta \boldsymbol{d}^{T}\left[\int_{\Omega^{-}} \boldsymbol{B}_{\tau-}^{T}[\boldsymbol{T}] \boldsymbol{B}_{\tau-} d A\right] \boldsymbol{d},
\end{aligned}
$$

where $\left\{\tau^{0}\right\}$, the initial surface stress vector, and $[\boldsymbol{T}]$, the surface elastic tensor, are defined by

$$
\left\{\tau^{0}\right\}=\left\{\begin{array}{l}
\tau_{11}^{0} \\
\tau_{22}^{0} \\
\tau_{12}^{0}
\end{array}\right\}, \quad[\boldsymbol{T}]=\left[\begin{array}{lll}
T_{1111} & T_{1122} & T_{1112} \\
T_{2211} & T_{2222} & T_{2212} \\
T_{1211} & T_{1222} & T_{1212}
\end{array}\right] .
$$

If the material and surface orientations of both the top $\left(\Omega^{+}\right)$and bottom $\left(\Omega^{-}\right)$surfaces are the same, (31) can be rewritten in terms of $\boldsymbol{B}_{m}$ and $\boldsymbol{B}_{b}$ from (17), as follows:

$$
\delta U_{\text {surf }}=\delta \boldsymbol{d}^{T}\left[\int_{\Omega} 2 \boldsymbol{B}_{m}^{T}\left\{\tau^{0}\right\} d A\right]+\delta \boldsymbol{d}^{T}\left[\int_{\Omega} 2 \boldsymbol{B}_{m}^{T}[\boldsymbol{T}] \boldsymbol{B}_{m} d A\right] \boldsymbol{d}+\delta \boldsymbol{d}^{T}\left[\int_{\Omega} \frac{h^{2}}{2} \boldsymbol{B}_{b}^{T}[\boldsymbol{T}] \boldsymbol{B}_{b} d A\right] \boldsymbol{d} .
$$

Combining (27) and (33) yields the following equation for internal virtual work, assuming the same surface type on both top and bottom surfaces:

$$
\begin{aligned}
\delta U=\delta \boldsymbol{d}^{T}\left[\int_{\Omega} \boldsymbol{B}_{m}^{T}\left[\boldsymbol{A}^{\prime}\right] \boldsymbol{B}_{m}+\right. & \left.\boldsymbol{B}_{b}^{T}\left[\boldsymbol{D}^{\prime}\right] \boldsymbol{B}_{b} d A\right] \boldsymbol{d}+\delta \boldsymbol{d}^{T}\left[\int_{\Omega} k \boldsymbol{B}_{s}^{T}[\boldsymbol{G}] \boldsymbol{B}_{s} d A\right] \boldsymbol{d} \\
& +\delta \boldsymbol{d}^{T}\left[\int_{\Omega} \boldsymbol{B}_{m}^{T}[\boldsymbol{B}] \boldsymbol{B}_{b}+\boldsymbol{B}_{b}^{T}[\boldsymbol{B}] \boldsymbol{B}_{m} d A\right] \boldsymbol{d}+\delta \boldsymbol{d}^{T}\left[\int_{\Omega} 2 \boldsymbol{B}_{m}^{T}\left\{\tau^{0}\right\} d A\right],
\end{aligned}
$$

where

$$
\left[\boldsymbol{A}^{\prime}\right]=[\boldsymbol{A}]+2[\boldsymbol{T}]=h[\boldsymbol{Q}]+2[\boldsymbol{T}] \quad \text { and } \quad\left[\boldsymbol{D}^{\prime}\right]=[\boldsymbol{D}]+\frac{1}{2} h^{2}[\boldsymbol{T}]=\frac{1}{12} h^{3}[\boldsymbol{Q}]+\frac{1}{2} h^{2}[\boldsymbol{T}]
$$

are respectively the effective membrane and effective bending rigidities. 


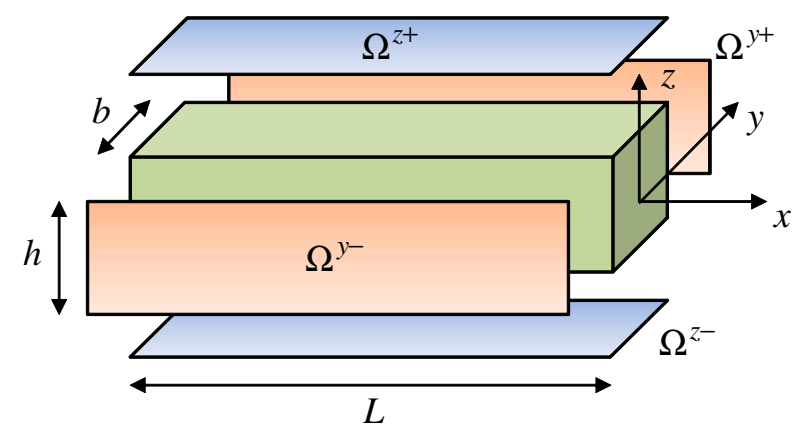

Figure 2. Schematic diagram of a rectangular nanowire with four surfaces.

2.2. Multiscale continuum model for a nanowire. To develop a model for a nanowire, a simple beam model with a rectangular cross-section is considered. This model accounts for both bending and shear deformations, but not for torsional effect. In Figure 2, $x$ is the axial direction, and $y$ and $z$ represent two perpendicular directions of the rectangular cross-section. Under the assumptions set forth by Timoshenko beam theory, the following displacement field is used:

$$
\begin{aligned}
& u_{x}=u_{x}^{0}+y \psi_{y}+z \psi_{z} \\
& u_{y}=u_{y}^{0}=v \\
& u_{z}=u_{z}^{0}=w
\end{aligned}
$$

The strain field is defined as

$$
\begin{aligned}
& \epsilon_{x x}=u_{x, x}^{0}+y \psi_{y, x}+z \psi_{z, x}, \\
& \gamma_{x y}=v_{, x}+\psi_{y}, \\
& \gamma_{x z}=w_{, x}+\psi_{z} .
\end{aligned}
$$

The displacements on the $y$-surface are given as

$$
\begin{aligned}
& u_{x}^{y \pm}=u_{x}^{0} \pm \frac{b}{2} \psi_{y}+z \psi_{z}, \\
& u_{y}^{y \pm}=v, \\
& u_{z}^{y \pm}=w,
\end{aligned}
$$

where $y+$ and $y-$ represent the top and bottom surfaces normal to the $y$-direction, and $b$ is the beam width along the $y$-direction. Similarly, the displacement on the $z$-surface can be written as follows:

$$
\begin{aligned}
u_{x}^{z^{ \pm}} & =u_{x}^{0}+y \psi_{y} \pm \frac{h}{2} \psi_{z}, \\
u_{y}^{z^{ \pm}} & =v, \\
u_{z}^{z \pm} & =w,
\end{aligned}
$$

where $z+$ and $z$ - represent the top and bottom surfaces normal to the $z$-direction, and $h$ is the beam thickness along the $z$-direction. 
According to the virtual work principle, the internal virtual work is equal to the external virtual work. The external virtual work is defined as

$$
\delta W_{E}=\int_{L} p_{x} \delta u_{x}^{0}+p_{y} \delta v+p_{z} \delta w d x
$$

where $p_{x}, p_{y}$, and $p_{z}$ are external forces in the $x, y$, and $z$ directions, respectively. The internal virtual work includes both bulk and surface energy components. The internal virtual work of the bulk component can be written as

$$
\begin{aligned}
\delta U_{\mathrm{bulk}}= & \int_{V} \sigma_{i j} \delta \epsilon_{i j} d V \\
= & \int_{V} \sigma_{x x} \delta \epsilon_{x x} d V+\int_{V} \sigma_{x y} \delta \gamma_{x y} d V+\int_{V} \sigma_{x z} \delta \gamma_{x z} d V \\
= & \int_{L} E_{x} A u_{x, x}^{0} \delta u_{x, x}^{0}+E_{x} I_{y y} \psi_{y, x} \delta \psi_{y, x}+E_{x} I_{z z} \psi_{z, x} \delta \psi_{z, x} d x \\
& \quad+\int_{L} k G_{66} A\left(\psi_{y}+v_{, x}\right) \delta\left(\psi_{y}+v_{, x}\right) d x+\int_{L} k G_{55} A\left(\psi_{z}+w_{, x}\right) \delta\left(\psi_{z}+w_{, x}\right) d x,
\end{aligned}
$$

where $E_{x}$ is the Young's modulus, $k$ is the shear correction factor, $A$ is the cross-sectional area, and $I_{y y}$ and $I_{z z}$ comprise the second moment of area, defined as

$$
I_{y y}=\int_{A} y^{2} d A, \quad I_{z z}=\int_{A} z^{2} d A .
$$

In the multiscale continuum model for a nanofilm, there are only two surfaces, top and bottom, whereas a nanowire with a rectangular cross-section has four surfaces: two in the $y$-direction $\left(\Omega^{y+}\right.$ and $\left.\Omega^{y-}\right)$ and two in the $z$-direction $\left(\Omega^{z+}\right.$ and $\Omega^{z-}$ ). The internal virtual work of these four surfaces is calculated as

$$
\begin{aligned}
\delta U_{\text {surf }}= & \int_{\Omega^{y \pm}}\left(\tau_{x x}^{y \pm} \delta \epsilon_{x x}^{y \pm}+\tau_{x z}^{y \pm} \delta \gamma_{x z}^{y \pm}\right) d \Omega^{y \pm}+\int_{\Omega^{z \pm}}\left(\tau_{x x}^{z \pm} \delta \epsilon_{x x}^{z \pm}+\tau_{x y}^{z \pm} \delta \gamma_{x y}^{z \pm}\right) d \Omega^{z \pm} \\
= & \int_{\Omega^{y \pm}} \tau_{x x}^{y \pm} \delta\left(u_{x, x}^{0} \pm \frac{b}{2} \psi_{y, x}+z \psi_{z . x}\right)+\tau_{x z}^{y \pm} \delta\left(\psi_{z}+w_{, x}\right) d \Omega^{y \pm} \\
& +\int_{\Omega^{z \pm}} \tau_{x x}^{z \pm} \delta\left(u_{x, x}^{0}+y \psi_{y, x} \pm \frac{h}{2} \psi_{z, x}\right)+\tau_{x y}^{z \pm} \delta\left(\psi_{y}+v_{, x}\right) d \Omega^{z \pm}
\end{aligned}
$$

where $\Omega$ is the surface domain, $\tau_{\alpha \beta}$ is the surface stress, and superscript $y \pm$ and $z \pm$ represent the $y$ surface and $z$-surface, respectively. Using linear surface elasticity, defined as

$$
\tau_{\alpha \beta}^{s}=\tau_{\alpha \beta}^{s 0}+T_{\alpha \beta \kappa \lambda}^{s} \epsilon_{\kappa \lambda}^{s \pm},
$$

the surface internal virtual work can be divided into two parts: the initial surface stress $\delta U_{\text {surf }}^{(\tau)}$ and the surface elastic tensor $\delta U_{\text {surf }}^{(T)}$ :

$$
\delta U_{\text {surf }}=\delta U_{\text {surf }}^{(\tau)}+\delta U_{\text {surf }}^{(T)} .
$$


Their values are

$$
\begin{aligned}
\delta U_{\text {surf }}^{(\tau)}= & \int_{\Omega^{y \pm}} \tau_{x x}^{y 0} \delta\left(u_{x, x}^{0} \pm \frac{b}{2} \psi_{y, x}+z \psi_{z, x}\right)+\tau_{x z}^{y 0} \delta\left(\psi_{z}+w_{, x}\right) d \Omega^{y \pm} \\
& +\int_{\Omega^{z \pm}} \tau_{x x}^{z 0} \delta\left(u_{x, x}^{0}+y \psi_{y, x} \pm \frac{h}{2} \psi_{z, x}\right)+\tau_{x y}^{z 0} \delta\left(\psi_{y}+v_{, x}\right) d \Omega^{z \pm}
\end{aligned}
$$

and

$$
\begin{aligned}
\delta U_{\text {surf }}^{(T)}= & \int_{\Omega^{y \pm}} T_{1111}^{y}\left(u_{x, x}^{0} \pm \frac{b}{2} \psi_{y, x}+z \psi_{z, x}\right) \delta\left(u_{x, x}^{0} \pm \frac{b}{2} \psi_{y, x}+z \psi_{z, x}\right) d \Omega^{y \pm} \\
& +\int_{\Omega^{y \pm}} T_{1212}^{y}\left(\psi_{z}+w_{, x}\right) \delta\left(\psi_{z}+w_{, x}\right) d \Omega^{y \pm} \\
& +\int_{\Omega^{z \pm}} T_{1111}^{z}\left(u_{x, x}^{0}+y \psi_{y, x} \pm \frac{h}{2} \psi_{z, x}\right) \delta\left(u_{x, x}^{0}+y \psi_{y, x} \pm \frac{h}{2} \psi_{z, x}\right) d \Omega^{z \pm} \\
& +\int_{\Omega^{z \pm}} T_{1212}^{z}\left(\psi_{y}+v_{, x}\right) \delta\left(\psi_{y}+v_{, x}\right) d \Omega^{z \pm} .
\end{aligned}
$$

Assuming that both the top and bottom surfaces in the $y$-direction are of the same type $\left(\Omega^{y+}=\right.$ $\left.\Omega^{y-}\right)$ and that both surfaces in the $z$-direction are also the same $\left(\Omega^{z+}=\Omega^{z-}\right)$, the initial surface stress component of the internal virtual work in (45) yields

$$
\begin{aligned}
\delta U_{\text {surf }}^{(\tau)}= & \int_{\Omega^{y}} 2 \tau_{x x}^{y 0} \delta\left(u_{x, x}^{0}+z \psi_{z, x}\right)+2 \tau_{x z}^{y 0} \delta\left(\psi_{z}+w_{, x}\right) d \Omega^{y} \\
& +\int_{\Omega^{z}} 2 \tau_{x x}^{z 0} \delta\left(u_{x, x}^{0}+y \psi_{y, x}\right)+2 \tau_{x y}^{z 0} \delta\left(\psi_{y}+v_{, x}\right) d \Omega^{z}
\end{aligned}
$$

and hence

$$
\delta U_{\text {surf }}^{(\tau)}=\int_{L} 2 h \tau_{x x}^{y 0} \delta u_{x, x}^{0}+2 h \tau_{x z}^{y 0} \delta\left(\psi_{z}+w_{, x}\right) d x+\int_{L} 2 b \tau_{x x}^{z 0} \delta u_{x, x}^{0}+2 b \tau_{x y}^{z 0} \delta\left(\psi_{y}+v_{, x}\right) d x .
$$

The surface elastic tensor portion of the internal virtual work in (46) can be rewritten as

$$
\begin{aligned}
\delta U_{\text {surf }}^{(T)}= & \int_{\Omega^{y}} 2 T_{1111}^{y}\left(u_{x, x}^{0}+z \psi_{z, x}\right) \delta\left(u_{x, x}^{0}+z \psi_{z, x}\right)+2 T_{1111}^{y}\left(\frac{b}{2} \psi_{y, x}\right) \delta\left(\frac{b}{2} \psi_{y, x}\right) d \Omega^{y} \\
& +\int_{\Omega^{y}} 2 T_{1212}^{y}\left(\psi_{z}+w_{, x}\right) \delta\left(\psi_{z}+w_{, x}\right) d \Omega^{y} \\
& +\int_{\Omega^{z}} 2 T_{1111}^{z}\left(u_{x, x}^{0}+y \psi_{y, x}\right) \delta\left(u_{x, x}^{0}+y \psi_{y, x}\right)+2 T_{1111}^{z}\left(\frac{h}{2} \psi_{z, x}\right) \delta\left(\frac{h}{2} \psi_{z, x}\right) d \Omega^{z} \\
& +\int_{\Omega^{z}} 2 T_{1212}^{z}\left(\psi_{y}+v_{, x}\right) \delta\left(\psi_{y}+v_{, x}\right) d \Omega^{z}
\end{aligned}
$$

and hence 


$$
\begin{array}{rl}
\delta U_{\text {surf }}^{(T)}=\int_{L} & 2 h T_{1111}^{y}\left(u_{x, x}^{0}\right) \delta\left(u_{x, x}^{0}\right) d x \\
& +\int_{L} \frac{1}{6} h^{3} T_{1111}^{y}\left(\psi_{z, x}\right) \delta\left(\psi_{z, x}\right)+\frac{1}{2} h b^{2} T_{1111}^{y}\left(\psi_{y, x}\right) \delta\left(\psi_{y, x}\right) d x \\
& +\int_{L} 2 h T_{1212}^{y}\left(\psi_{z}+w_{, x}\right) \delta\left(\psi_{z}+w_{, x}\right) d x \\
& +\int_{L} 2 b T_{1111}^{z}\left(u_{x, x}^{0}\right) \delta\left(u_{x, x}^{0}\right) d x \\
& +\int_{L} \frac{1}{6} b^{3} T_{1111}^{z}\left(\psi_{y, x}\right) \delta\left(\psi_{y, x}\right)+\frac{1}{2} b h^{2} T_{1111}^{z}\left(\psi_{z, x}\right) \delta\left(\psi_{z, x}\right) d x \\
& +\int_{L} 2 b T_{1212}^{z}\left(\psi_{y}+v_{, x}\right) \delta\left(\psi_{y}+v_{, x}\right) d x .
\end{array}
$$

Finite element implementation for a nanowire. The displacement field of a nanowire can be interpolated using an interpolation function $N_{i}$ and the nodal displacement

$$
\boldsymbol{d}=\left[\left(u_{x}^{0}, v, w, \psi_{y}, \psi_{z}\right)_{1}, \ldots,\left(u_{x}^{0}, v, w, \psi_{y}, \psi_{z}\right)_{n}\right]^{T}
$$

so that

$$
u_{x}^{0}=\sum_{i=1}^{n} N_{i} u_{x i}^{0}, \quad v=\sum_{i=1}^{n} N_{i} v_{i}, \quad w=\sum_{i=1}^{n} N_{i} w_{i}, \quad \psi_{y}=\sum_{i=1}^{n} N_{i} \psi_{y i}, \quad \psi_{z}=\sum_{i=1}^{n} N_{i} \psi_{z i}
$$

where $n$ is the number of nodes per element. Using these nodal displacements, the membrane aspect of the bulk internal virtual work can be rewritten as

$$
\delta U_{\mathrm{bulk}}^{\mathrm{mem}}=\int_{L} E_{x} A u_{x, x}^{0} \delta u_{x, x}^{0} d x=\delta \boldsymbol{d}^{T}\left[\int_{L} \boldsymbol{B}_{m}^{T}[\boldsymbol{A}] \boldsymbol{B}_{m} d x\right] \boldsymbol{d},
$$

where

$$
\begin{gathered}
{[\boldsymbol{A}]=\left[E_{x} A\right],} \\
\left\{u_{x, x}^{0}\right\}=\boldsymbol{B}_{m} \boldsymbol{d}=\left[\boldsymbol{B}_{m}^{(1)}, \boldsymbol{B}_{m}^{(2)}, \ldots, \boldsymbol{B}_{m}^{(n)}\right] \boldsymbol{d}, \\
\boldsymbol{B}_{m}^{(i)}=\left[\begin{array}{lllll}
N_{i, x} & 0 & 0 & 0 & 0
\end{array}\right] .
\end{gathered}
$$

The bending component of the internal virtual work is defined as

$$
\begin{aligned}
\delta U_{\mathrm{bulk}}^{\mathrm{bend}} & =\int_{L} E_{x} I_{y y} \psi_{y, x} \delta \psi_{y, x}+E_{x} I_{z z} \psi_{z, x} \delta \psi_{z, x} d x \\
& =\delta \boldsymbol{d}^{T}\left[\int_{L} \boldsymbol{B}_{b}^{T}[\boldsymbol{D}] \boldsymbol{B}_{b} d x\right] \boldsymbol{d},
\end{aligned}
$$


where

$$
\begin{gathered}
{[\boldsymbol{D}]=\left[\begin{array}{cc}
E_{x} I_{y y} & 0 \\
0 & E_{x} I_{z z}
\end{array}\right],} \\
\left\{\begin{array}{l}
\psi_{y, x} \\
\psi_{z, x}
\end{array}\right\}=\boldsymbol{B}_{b} \boldsymbol{d}=\left[\boldsymbol{B}_{b}^{(1)}, \boldsymbol{B}_{b}^{(2)}, \ldots, \boldsymbol{B}_{b}^{(n)}\right] \boldsymbol{d}, \\
\boldsymbol{B}_{b}^{(i)}=\left[\begin{array}{ccccc}
0 & 0 & 0 & N_{i, x} & 0 \\
0 & 0 & 0 & 0 & N_{i, x}
\end{array}\right] .
\end{gathered}
$$

The transverse shear component of the virtual work is

$$
\begin{aligned}
\delta U_{\text {bulk }}^{\text {shear }} & =\int_{L} k G_{66} A\left(\psi_{y}+v_{, x}\right) \delta\left(\psi_{y}+v_{, x}\right) d x+\int_{L} k G_{55} A\left(\psi_{z}+w_{, x}\right) \delta\left(\psi_{z}+w_{, x}\right) d x \\
& =\delta \boldsymbol{d}^{T}\left[\int_{L} k \boldsymbol{B}_{s}^{T}[\boldsymbol{G}] \boldsymbol{B}_{s} d x\right] \boldsymbol{d},
\end{aligned}
$$

where

$$
\begin{gathered}
{[\boldsymbol{G}]=\left[\begin{array}{cc}
G_{66} & 0 \\
0 & G_{55}
\end{array}\right],} \\
\left\{\begin{array}{l}
v_{, x}+\psi_{y} \\
\left.w_{, x}+\psi_{z}\right\}
\end{array}\right\}=\boldsymbol{B}_{s} \boldsymbol{d}=\left[\boldsymbol{B}_{s}^{(1)}, \boldsymbol{B}_{s}^{(2)}, \ldots, \boldsymbol{B}_{s}^{(n)}\right] \boldsymbol{d}, \\
\boldsymbol{B}_{s}^{(i)}=\left[\begin{array}{ccccc}
0 & N_{i, x} & 0 & N_{i} & 0 \\
0 & 0 & N_{i, x} & 0 & N_{i}
\end{array}\right] .
\end{gathered}
$$

The internal virtual work of the bulk component can be obtained with a summation of Equations (52), (56), and (60):

$$
\delta U_{\text {bulk }}=\delta \boldsymbol{d}^{T}\left[\int_{L} \boldsymbol{B}_{m}^{T}[\boldsymbol{A}] \boldsymbol{B}_{m}+\boldsymbol{B}_{b}^{T}[\boldsymbol{D}] \boldsymbol{B}_{b}+k \boldsymbol{B}_{s}^{T}[\boldsymbol{G}] \boldsymbol{B}_{s} d x\right] \boldsymbol{d} .
$$

As for surface internal virtual work, the initial surface stress component of the internal virtual work in (48) can be written in the following discrete form:

$$
\delta U_{\text {surf }}^{(\tau)}=\int_{L}\left\{\begin{array}{c}
u_{x, x}^{0} \\
\psi_{y}+v_{, x} \\
\psi_{z}+w_{, x}
\end{array}\right\}^{T}\left\{\begin{array}{c}
2 h \tau_{x x}^{y 0}+2 b \tau_{x x}^{z 0} \\
2 b \tau_{x y}^{z 0} \\
2 h \tau_{x z}^{y 0}
\end{array}\right\} d x=\delta \boldsymbol{d}^{T}\left[\int_{L} \boldsymbol{B}_{\tau}^{T}\left\{\tau^{0}\right\} d x\right]
$$

where

$$
\begin{gathered}
\left\{\tau^{0}\right\}=\left\{\begin{array}{c}
2 h \tau_{x x}^{y 0}+2 b \tau_{x x}^{z 0} \\
2 b \tau_{x y}^{z 0} \\
2 h \tau_{x z}^{y 0}
\end{array}\right\}, \\
\left\{\begin{array}{c}
u_{x, x}^{0} \\
\psi_{y}+v_{, x} \\
\psi_{z}+w_{, x}
\end{array}\right\}=\boldsymbol{B}_{\tau} \boldsymbol{d}=\left[\boldsymbol{B}_{\tau}^{(1)}, \boldsymbol{B}_{\tau}^{(2)}, \ldots, \boldsymbol{B}_{\tau}^{(n)}\right] \boldsymbol{d},
\end{gathered}
$$


and

$$
\boldsymbol{B}_{\tau}^{(i)}=\left[\begin{array}{ccccc}
N_{i, x} & 0 & 0 & 0 & 0 \\
0 & N_{i, x} & 0 & N_{i} & 0 \\
0 & 0 & N_{i, x} & 0 & N_{i}
\end{array}\right]
$$

The surface elastic tensor component of the internal virtual work in (50) can be expressed in the following discrete form, under the assumption that $\Omega^{y+}=\Omega^{y-}$ and $\Omega^{z+}=\Omega^{z-}$ :

$$
\begin{aligned}
\delta U_{\text {surf }}^{(T)}= & \int_{L}\left(2 h T_{1111}^{y}+2 b T_{1111}^{z}\right)\left(u_{x, x}^{0}\right) \delta\left(u_{x, x}^{0}\right) d x \\
& +\int_{L}\left(\frac{1}{6} h^{3} T_{1111}^{y}+\frac{1}{2} b h^{2} T_{1111}^{z}\right)\left(\psi_{z, x}\right) \delta\left(\psi_{z, x}\right) d x \\
& +\int_{L}\left(\frac{1}{6} b^{3} T_{1111}^{z}+\frac{1}{2} h b^{2} T_{1111}^{z}\right)\left(\psi_{y, x}\right) \delta\left(\psi_{y, x}\right) d x \\
& +\int_{L} 2 h T_{1212}^{y}\left(\psi_{z}+w_{, x}\right) \delta\left(\psi_{z}+w_{, x}\right) d x \\
& +\int_{L} 2 b T_{1212}^{z}\left(\psi_{y}+v_{, x}\right) \delta\left(\psi_{y}+v_{, x}\right) d x \\
= & \delta \boldsymbol{d}^{T}\left[\int_{L} \boldsymbol{B}_{m}^{T}\left[\boldsymbol{T}_{m}\right] \boldsymbol{B}_{m}+\boldsymbol{B}_{b}^{T}\left[\boldsymbol{T}_{b}\right] \boldsymbol{B}_{b}+\boldsymbol{B}_{s}^{T}\left[\boldsymbol{T}_{s}\right] \boldsymbol{B}_{s} d x\right] \boldsymbol{d},
\end{aligned}
$$

where

$$
\begin{aligned}
& {\left[\boldsymbol{T}_{m}\right]=\left[2 h T_{1111}^{y}+2 b T_{1111}^{z}\right],} \\
& {\left[\boldsymbol{T}_{b}\right]=\left[\begin{array}{cc}
\frac{1}{2} b^{2} h T_{1111}^{y}+\frac{1}{6} b^{3} T_{1111}^{z} & 0 \\
0 & \frac{1}{6} h^{3} T_{1111}^{y}+\frac{1}{2} b h^{2} T_{1111}^{z}
\end{array}\right],} \\
& {\left[\boldsymbol{T}_{s}\right]=\left[\begin{array}{cc}
2 b T_{1212}^{z} & 0 \\
0 & 2 h T_{1212}^{y}
\end{array}\right],}
\end{aligned}
$$

and

$$
\begin{array}{r}
\left\{u_{x, x}^{0}\right\}=\boldsymbol{B}_{m} \boldsymbol{d}=\left[\boldsymbol{B}_{m}^{(1)}, \boldsymbol{B}_{m}^{(2)}, \ldots, \boldsymbol{B}_{m}^{(n)}\right] \boldsymbol{d}, \\
\left\{\begin{array}{l}
\psi_{y, x} \\
\psi_{z, x}
\end{array}\right\}=\boldsymbol{B}_{b} \boldsymbol{d}=\left[\boldsymbol{B}_{b}^{(1)}, \boldsymbol{B}_{b}^{(2)}, \ldots, \boldsymbol{B}_{b}^{(n)}\right] \boldsymbol{d}, \\
\left\{\begin{array}{c}
v_{, x}+\psi_{y} \\
w_{, x}+\psi_{z}
\end{array}\right\}=\boldsymbol{B}_{s} \boldsymbol{d}=\left[\boldsymbol{B}_{s}^{(1)}, \boldsymbol{B}_{s}^{(2)}, \ldots, \boldsymbol{B}_{s}^{(n)}\right] \boldsymbol{d},
\end{array}
$$

with

$$
\begin{aligned}
\boldsymbol{B}_{m}^{(i)} & =\left[\begin{array}{lllll}
N_{i, x} & 0 & 0 & 0 & 0
\end{array}\right], \\
\boldsymbol{B}_{b}^{(i)} & =\left[\begin{array}{lllll}
0 & 0 & 0 & N_{i, x} & 0 \\
0 & 0 & 0 & 0 & N_{i, x}
\end{array}\right], \\
\boldsymbol{B}_{s}^{(i)} & =\left[\begin{array}{ccccc}
0 & N_{i, x} & 0 & N_{i} & 0 \\
0 & 0 & N_{i, x} & 0 & N_{i}
\end{array}\right] .
\end{aligned}
$$


From (65) and (69), the matrix form of the surface internal virtual work can be written as

$$
\delta U_{\text {surf }}=\delta \boldsymbol{d}^{T}\left[\int_{L} \boldsymbol{B}_{m}^{T}\left[\boldsymbol{T}_{m}\right] \boldsymbol{B}_{m}+\boldsymbol{B}_{b}^{T}\left[\boldsymbol{T}_{b}\right] \boldsymbol{B}_{b}+\boldsymbol{B}_{s}^{T}\left[\boldsymbol{T}_{s}\right] \boldsymbol{B}_{s} d x\right] \boldsymbol{d}+\delta \boldsymbol{d}^{T}\left[\int_{L} \boldsymbol{B}_{\tau}^{T}\left\{\tau^{0}\right\} d x\right] .
$$

Consequently, the following matrix form of the internal virtual work is obtained for a nanowire with a rectangular cross-section:

$$
\delta U=\delta \boldsymbol{d}^{T}\left[\int_{L} \boldsymbol{B}_{m}^{T}\left[\boldsymbol{A}^{\prime}\right] \boldsymbol{B}_{m}+\boldsymbol{B}_{b}^{T}\left[\boldsymbol{D}^{\prime}\right] \boldsymbol{B}_{b}+k \boldsymbol{B}_{s}^{T}\left[\boldsymbol{G}^{\prime}\right] \boldsymbol{B}_{s} d x\right] \boldsymbol{d}+\delta \boldsymbol{d}^{T}\left[\int_{L} \boldsymbol{B}_{\tau}^{T}\left\{\tau^{0}\right\} d x\right],
$$

where

$$
\begin{aligned}
{\left[\boldsymbol{A}^{\prime}\right] } & =\left[\boldsymbol{A}\left(E_{1}+\frac{2}{b} T_{1111}^{y}+\frac{2}{h} T_{1111}^{z}\right)\right], \\
{\left[\boldsymbol{D}^{\prime}\right] } & =\left[\begin{array}{cc}
I_{y y}\left(E_{1}+\frac{6}{b} T_{1111}^{y}+\frac{2}{h} T_{1111}^{z}\right) & 0 \\
0 & I_{z z}\left(E_{1}+\frac{2}{b} T_{1111}^{y}+\frac{6}{h} T_{1111}^{z}\right)
\end{array}\right], \\
{\left[\boldsymbol{G}^{\prime}\right] } & =\left[\begin{array}{cc}
A\left(G_{66}+\frac{2}{h} T_{1212}^{z}\right) & 0 \\
0 & A\left(G_{55}+\frac{2}{b} T_{1212}^{y}\right)
\end{array}\right] .
\end{aligned}
$$

\section{Elastic modulus and surface elastic tensor}

In this study, an MD simulation is performed to calculate the elastic properties of bulk material. FCC single crystal copper is used as a test material. The LAMMPS software (http://lammps.sandia.gov) [Plimpton 1995] and the embedded atom method (EAM) [Daw and Baskes 1984; Foiles et al. 1986] are utilized for the MD simulation. Four nanofilms and three nanowires of different crystallographic orientations are investigated: $\{100\} /<100>,\{100\} /<110>,\{111\} /<110>$, and $\{110\} /<100>$ nanofilms, and $\langle 100>/\{100\},<100>/\{110\}$, and $<110>/\{100\}$ nanowires.

3.1. Elastic modulus of the bulk material. In order to calculate the elastic constants of single-crystal copper, a $4 \times 4 \times 4$ unit cell with periodic boundary conditions (PBCs) [Rapaport 2004] is utilized. When a periodic boundary condition is applied, a particle that moves to the right will reappear on the left, as shown in Figure 3(c). PBCs are applied to all directions in the bulk simulation. For initial relaxation, the MD simulations are performed under an isothermal-isobaric ensemble (where NPT represents a constant number of atoms, constant pressure, and a constant temperature) at $0.1 \mathrm{~K}$ for 200 picoseconds. After that, the simulation box is deformed slowly, via a $0.01 \%$ applied strain in the 1-direction, under the canonical ensemble (where NVT denotes a constant number of atoms, constant volume, and constant temperature) over a span of 50 picoseconds, as shown in Figure 3(a). Next, a 50-picosecond MD simulation is performed to get the average values of the virial stresses, which are defined as

$$
\sigma_{i j}^{v}=\frac{1}{V_{0}} \sum_{\alpha}\left[-m^{\alpha} v_{i}^{\alpha} v_{i}^{\beta}+\frac{1}{2} \sum_{\beta=1}^{N}\left(R_{i}^{\beta}-R_{i}^{\alpha}\right) F_{j}^{\alpha \beta}\right],
$$




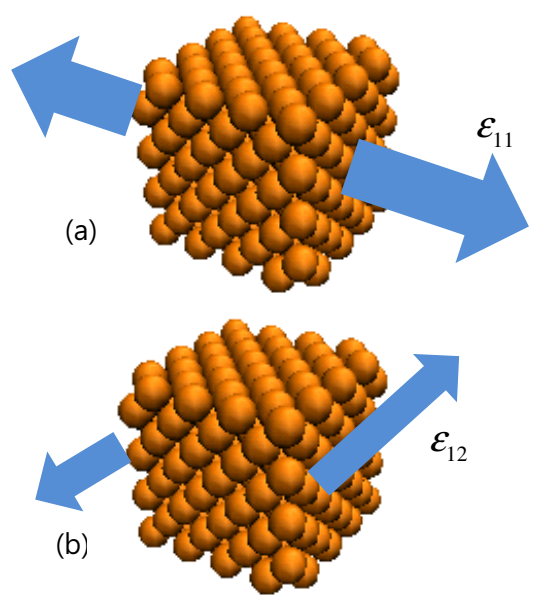

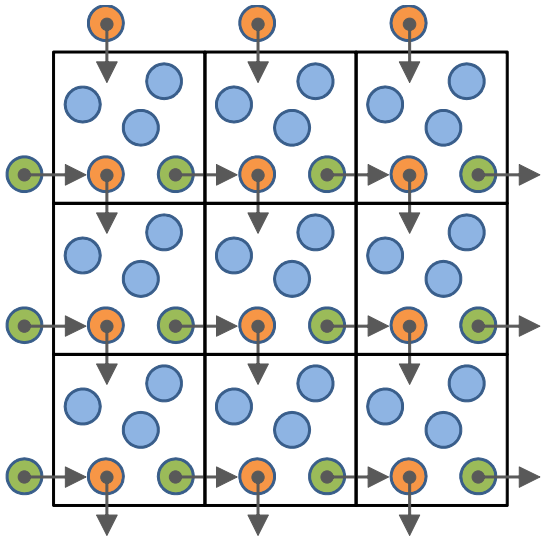

(c) Periodic Boundary Condition

Figure 3. Molecular dynamics simulation for the calculation of elastic constants: (a) depiction of normal strain; (b) depiction of shear strain; (c) schematic of periodic boundary condition.

where $\sigma_{i j}^{v}$ is a component of virial stress, $V_{0}$ is the volume of the simulation box, $m^{\alpha}$ is the mass, $v_{i}^{\alpha}$ is the $i$-directional velocity component, $R_{i}^{\alpha}$ is the $i$-directional position of atom $\alpha$, and $F_{j}^{\alpha \beta}$ is the $j$ directional interatomic force between atoms $\alpha$ and $\beta$ [Subramaniyan and Sun 2008]. The same procedure is performed under shear deformation for a shear component (see Figure 3(b)). As a result of these simulations, the material constants $C_{11}, C_{12}$, and $C_{44}$ are calculated using the linear constitutive relationship $\sigma_{i}^{v}=C_{i j} \epsilon_{j}$ :

$$
\begin{aligned}
\sigma_{1}^{v} & =C_{11} \hat{\epsilon}_{1}, \\
\sigma_{2}^{v} & =C_{12} \hat{\epsilon}_{1}, \\
\sigma_{4}^{v} & =C_{44} \hat{\epsilon}_{4},
\end{aligned}
$$

where $\sigma_{i}^{v}, C_{i j}$, and $\hat{\epsilon}_{i}$ are virial stress, elastic constant, and applied strain in Voigt notation, respectively. The elastic constants of the bulk material are listed in Voigt form in Table 1. The elastic constants of the $\{100\} /<100>$ direction are obtained from the MD simulation, and those of the other directions are calculated via coordinate transformations based on the $C_{11}, C_{12}$, and $C_{44}$ values of the $\{100\} /<100>$ direction.

The $\{100\} /<100>$ nanofilm features $\{100\}$ surfaces on the top and bottom. The two in-plane axes of the surface exist in the $<100>$ direction. In this case, the film's material properties have cubic symmetry. The Young's modulus value of the $\{100\} /<100>$ nanofilm is $61.5 \mathrm{GPa}$, and its Poisson's ratio is 0.43 .

The surface type of the $\{100\} /<110>$ nanofilm is also $\{100\}$, but its two in-plane axes are oriented in the $<110>$ direction. The values of Young's moduli in the in-plane direction $\left(E_{1}\right.$ and $\left.E_{2}\right)$ are $126.0 \mathrm{GPa}$, and the Poisson's ratios are $v_{12}=-0.18$ and $v_{13}=0.87$.

Being isotropic, the $\{111\}$ surface has uniform material properties in all in-plane directions. However, the $\{111\}$ nanofilm has nonzero values for $C_{14}, C_{24}$, and $C_{56}\left(C_{14}=-C_{24}=25.9 \mathrm{GPa}\right.$ and $C_{56}=$ 


\begin{tabular}{|ccccc|}
\hline & $\{100\} /<100>$ & $\{100\} /<110>$ & $\{111\} /<110>$ & $\{110\} /<100>>$ \\
$C_{11}$ & 167.3 & 222.1 & 222.1 & 167.3 \\
$C_{22}$ & 167.3 & 222.1 & 222.1 & 222.1 \\
$C_{33}$ & 167.3 & 167.3 & 240.4 & 222.1 \\
$C_{12}$ & 124.2 & 69.3 & 105.9 & 124.2 \\
$C_{13}$ & 124.2 & 124.2 & 87.6 & 124.2 \\
$C_{23}$ & 124.2 & 124.2 & 87.6 & 69.3 \\
$C_{44}$ & 76.4 & 76.4 & 39.9 & 21.6 \\
$C_{55}$ & 76.4 & 76.4 & 39.9 & 76.4 \\
$C_{66}$ & 76.4 & 21.6 & 58.1 & 76.4 \\
\hline
\end{tabular}

Table 1. Elastic constants of bulk copper film (in GPa).

25.9 GPa). These variables equal zero for all other orthotropic surfaces. The Young's modulus of the in-plane direction $\left(E_{1}\right)$ is $126.0 \mathrm{GPa}$, and its Poisson's ratio is $v_{12}=0.52$.

Unlike $\{100\}$ and $\{111\}$ surfaces, the $\{110\}$ surface is not axis-symmetric. The $\{110\} /<100>$ surface has two different in-plane axis orientations: $\langle 100\rangle$ for the 1-direction and $\langle 110\rangle$ for the 2-direction. The Young's moduli of these two orientations are $E_{1}=61.5 \mathrm{GPa}$ and $E_{2}=E_{3}=126.0 \mathrm{GPa}$, respectively, and their Poisson's ratios are $v_{12}=0.43$ and $v_{21}=0.87$.

As for copper nanowires, the Young's moduli of both $<100>/\{100\}$ and $<100>/\{110\}$ nanowires are $61.5 \mathrm{GPa}$, and the Young's modulus of a $<110>/\{100\}$ nanowire is $126.0 \mathrm{GPa}$.

3.2. Size-dependent elasticity and surface elastic tensor of copper nanofilms. For a nanoscaled thin film, mechanical properties such as Young's modulus and Poisson's ratio vary depending on the film thickness. An MD simulation is performed to evaluate the size-dependent elastic properties of copper nanofilms. Four types of MD model are used for the calculation of effective moduli, simulating nanofilms with $\{100\} /<100\rangle,\{100\} /<110\rangle,\{111\} /<110\rangle$, and $\{110\} /<100>$ surfaces.

For the MD nanofilm simulations, periodic boundary conditions (PBCs) are applied in both the 1and 2-directions. A free boundary condition is applied in the direction of the nanofilm thickness. As in the bulk case, relaxation and deformation processes are performed, and a two-dimensional constitutive equation is used. The initial lattice spacing of nanofilms is set by the lattice constant of the bulk material. The NPT ensemble is utilized in the first step of the MD simulation to get an equilibrium configuration. The equilibrium strain of a nanofilm is then obtained from this equilibrium configuration. After that, the virial stress under a given applied strain is calculated using the NVT ensemble.

The effective membrane stiffness tensor, $\left[\boldsymbol{A}^{\prime}\right]$, in (35) can be obtained from the relationship between the applied strain and the virial stress, which is calculated using the results of the MD simulation.

$$
\frac{1}{N_{\text {layer }}} \sum_{k=1}^{N_{\text {layer }}}\left\{\begin{array}{l}
\sigma_{11}^{v} \\
\sigma_{22}^{v} \\
\sigma_{12}^{v}
\end{array}\right\}^{(k)}=\frac{1}{h}\left[\boldsymbol{A}^{\prime}\right]\left\{\begin{array}{c}
\hat{\epsilon}_{11} \\
\hat{\epsilon}_{22} \\
2 \hat{\epsilon}_{12}
\end{array}\right\} \text {, }
$$

where $\sigma_{\alpha \beta}^{v^{(k)}}$ is the virial stress of atoms in the $k$-th layer, $\hat{\epsilon}_{\alpha \beta}$ is the applied strain, $N_{\text {layer }}$ is the number of layers, and $h$ is the film thickness. 


\begin{tabular}{|ccccc|}
\hline & $\{100\} /<100>$ & $\{100\} /<110>$ & $\{111\} /<110>$ & $\{110\} /<100>$ \\
$T_{1111}$ & 9.8084 & 13.7315 & 9.4804 & -15.0566 \\
$T_{2222}$ & 9.8084 & 13.7315 & 9.4804 & -2.2873 \\
$T_{1122}$ & 25.5585 & 5.3080 & 4.1747 & -5.1194 \\
$T_{1212}$ & 10.9082 & -5.4126 & 2.6531 & -1.0701 \\
\hline
\end{tabular}

Table 2. Calculated surface elastic tensor of nanofilms using the one-point matching method (in N/m).

Considering the symmetry, the total number of unknowns in the $3 \times 3$ matrix $\left[\boldsymbol{A}^{\prime}\right]$ is six. However, the number of unknowns of a $\{100\} /<100>$ film reduces to four, i.e. $A_{11}^{\prime}, A_{12}^{\prime}, A_{13}^{\prime}$, and $A_{33}^{\prime}$, because of the film's in-plane axis symmetry. Three of these components, $A_{11}^{\prime}, A_{12}^{\prime}$, and $A_{13}^{\prime}$, can be determined using stress-strain equations obtained from the MD simulation with an applied strain of $\hat{\epsilon}_{\alpha \beta}=\lfloor 0.01 \%, 0,0\rfloor^{T}$. The component $A_{33}^{\prime}$ is determined from the MD simulation with an applied strain of $\hat{\epsilon}_{\alpha \beta}=\lfloor 0,0,0.01 \%\rfloor^{T}$.

The $\left[\boldsymbol{A}^{\prime}\right]$ matrices for $\{100\} /<110>$ and $\{111\} /<110>$ films are determined in the same way. Three MD simulations are performed for the $\{110\} /<100>$ film, in order to collect sufficient data to calculate the components of matrix $\left[\boldsymbol{A}^{\prime}\right]$. An additional applied strain, $\hat{\epsilon}_{\alpha \beta}=\lfloor 0,0.01 \%, 0\rfloor^{T}$, is utilized to determine the $A_{12}^{\prime}, A_{22}^{\prime}$, and $A_{32}^{\prime}$ values of the $\{110\} /<100>$ nanofilm.

The surface elastic tensor $T_{\alpha \beta \kappa \lambda}$, a component of [T], can be calculated from (35) by applying a onepoint matching technique to the results from the MD simulation. In this calculation, the matrix $[\boldsymbol{Q}]$ in (35), which represents the two-dimensional constitutive relationship under the plane-stress condition, is determined from the bulk elastic constants given in (20).

The surface elastic tensor matrix $[\boldsymbol{T}]$ is a symmetric matrix with the components listed in Table 2 . The missing components in Table 2 are zeros $\left(T_{1112}=T_{2212}=0\right)$. According to the matching method, the MD simulations use $3.1 \mathrm{~nm}$-thick $\{100\} /<100>$ and $\{100\} /<110>$ nanofilms and $5.2 \mathrm{~nm}$ and $4.2 \mathrm{~nm}$-thick $\{111\} /<110>$ and $\{110\} /<100>$ nanofilms, respectively.

Figures 4 and 5 show size-dependent Young's moduli normalized by bulk material data. The results for $\{100\} /<100>,\{100\} /<110>$, and $\{111\} /<110>$ nanofilms are shown in Figure 4 . The in-plane axes of the $\{110\} /<100>$ nanofilm have two different orientations: $<100>$ and $<110>$. The relative moduli are denoted as E1 and E2, as shown in Figure 5. Size-dependent shear moduli normalized by bulk material data are shown in Figure 6. The lines are the results of the proposed continuum model and the marks represent the results of an MD simulation.

3.3. Size-dependent elasticity and surface elastic tensor of copper nanowires. When studying the sizedependent elasticity of a nanowire, three types of copper nanowires were considered: $<100>/\{100\}$, $<100>/\{110\}$, and $<110>/\{100\}$. Every nanowire has a rectangular cross-section and four surfaces. The $<100>/\{100\}$ nanowire has four $\{100\}$ surfaces, two in the $y$-direction and two in the $z$-direction, and the $<100>/\{110\}$ nanowire has four $\{110\}$ surfaces. The $<110>/\{100\}$ nanowire has two $\{100\}$ surfaces in the $y$-direction and two $\{110\}$ surfaces in the $z$-direction.

The elastic modulus of a nanowire is influenced by the surface elastic tensor of the $y$ - and $z$-surfaces, as described by (75). The surface elastic tensor of a nanowire is determined by applying the matching technique to the results of the MD simulation. In the cases involving $<100>/\{100\}$ and $<100>/\{110\}$ 


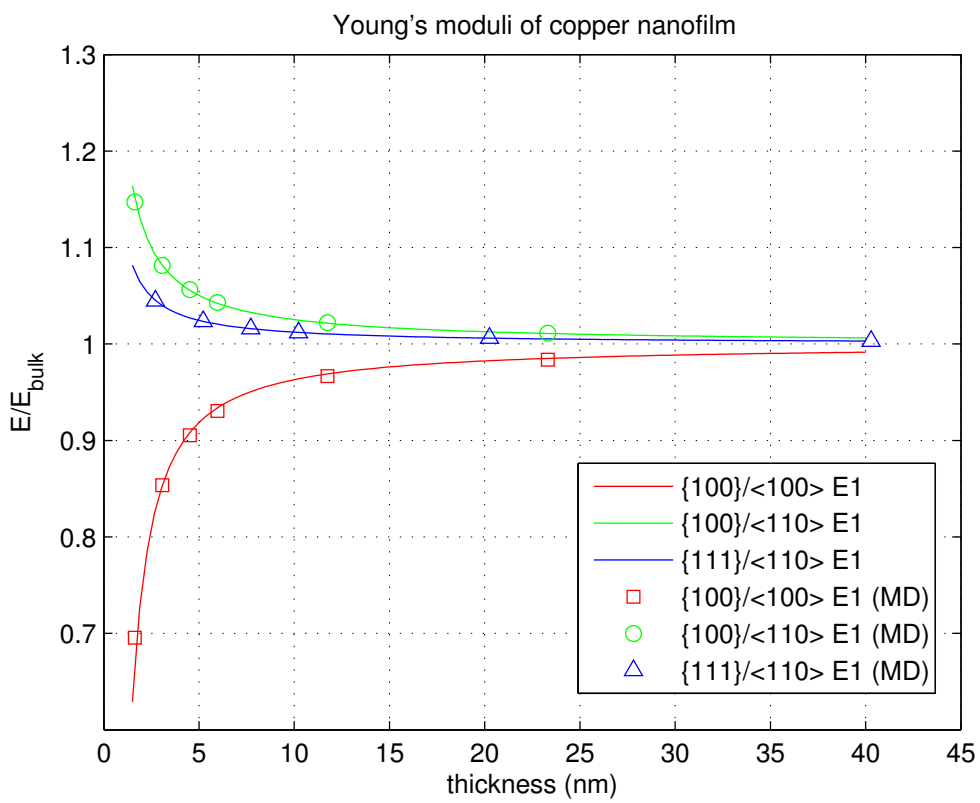

Figure 4. Size-dependent Young's moduli of $\{100\} /<100>,\{100\} /<110>,\{111\} /<110>$ copper nanofilms.

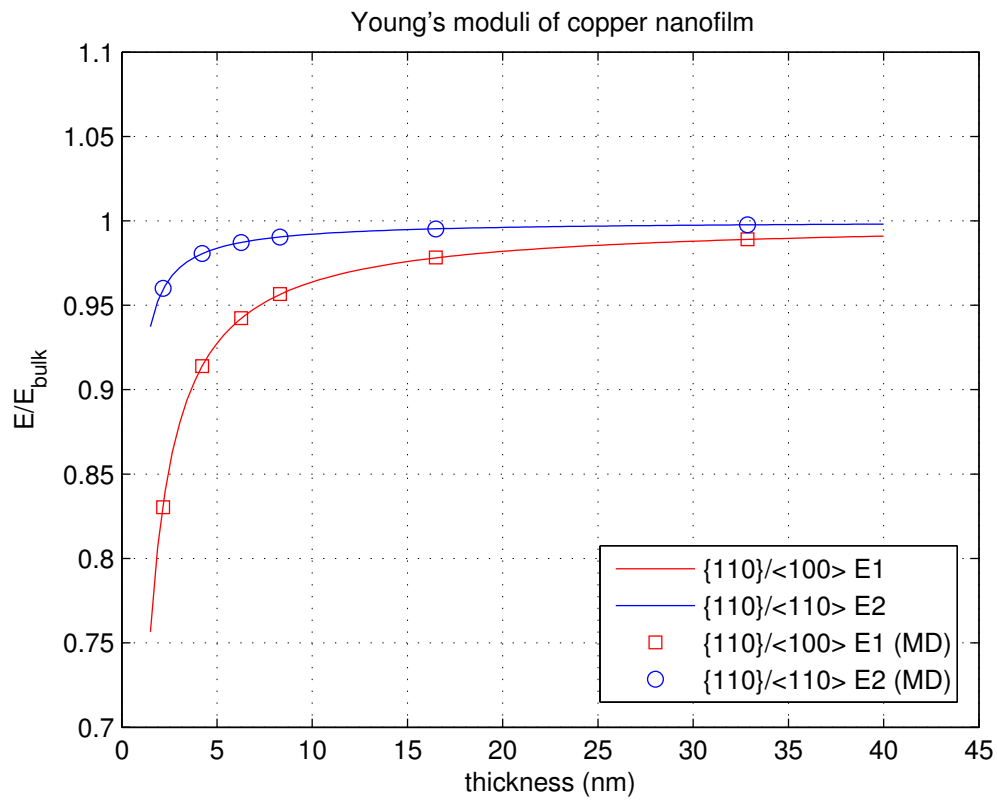

Figure 5. Size-dependent Young's moduli of a $\{110\} /<100>$ copper nanofilm.

nanowires, only one MD simulation is needed for one-point matching because the surfaces in the $y$ - and $z$-directions are identical. However, the $\langle 110>/\{100\}$ nanowire needs two MD simulations, one for the surface in the $y$-direction and one for the surface in the $z$-direction. 


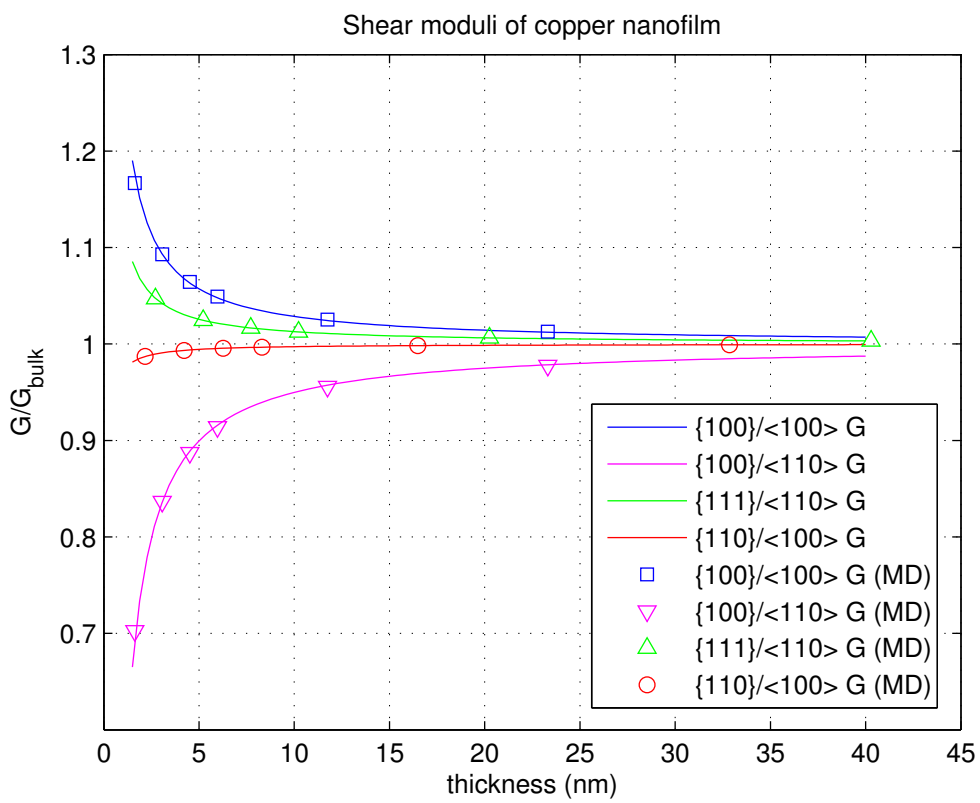

Figure 6. Size-dependent shear moduli of $\{100\} /<100\rangle,\{100\} /<110>,\{111\} /<110\rangle$, and $\{110\} /<100>$ nanofilms.

\begin{tabular}{|cccc|}
\hline & $<100>/\{100\}$ & $<100>/\{110\}$ & $<110>/\{100\}$ \\
$T_{111}^{y}$ & -10.7613 & -11.3434 & 27.3160 \\
$T_{1111}^{z}$ & -10.7613 & -11.3434 & -9.9535 \\
\hline
\end{tabular}

Table 3. Surface elastic tensor of nanowires calculated by the matching method (in N/m).

In the matching method, the dimension $b=h=4.5 \mathrm{~nm}$ is used for the $<100>/\{100\}$ nanowire, and $b=h=6.3 \mathrm{~nm}$ is set for the $\langle 100>/\{110\}$ nanowire. For the $<110>/\{100\}$ nanowire, the width is set to $b=4.5 \mathrm{~nm}$ in the first simulation and $b=8.9 \mathrm{~nm}$ in the second simulation, and the thickness for both simulations is fixed at $h=6.3 \mathrm{~nm}$.

The calculated surface elastic tensors for copper nanowires are listed in Table 3. As shown in Figure 7, the Young's moduli evaluated by the proposed continuum-based model (see the lines in the figure) have good agreement with the results of the MD simulation (see the marks in the figure). The "thickness" in Figure 7 represents the width, $b$, of a nanowire. In the $z$-direction, $h=b$ for $<100>/\{100\}$ and $<100>/\{110\}$ nanowires, and $h=\sqrt{2}(b-a / 4)$ for the $<110>/\{100\}$ nanowire, where $a$ is the lattice constant of FCC single crystal copper.

3.4. Numerical examples of beam vibration. As an example of a surface effect on a nanofilm, the natural frequencies of clamped beams are investigated using the finite element method. The mesh configuration and vibration modes of the clamped beam are shown in Figure 8. The length and width of the beams are fixed at $300 \mathrm{~nm}$ and $30 \mathrm{~nm}$, respectively, and the thickness varies from $3 \mathrm{~nm}$ to $12 \mathrm{~nm}$. Isoparametric 4-noded plate elements are used in the finite element analysis, and a selective reduced 


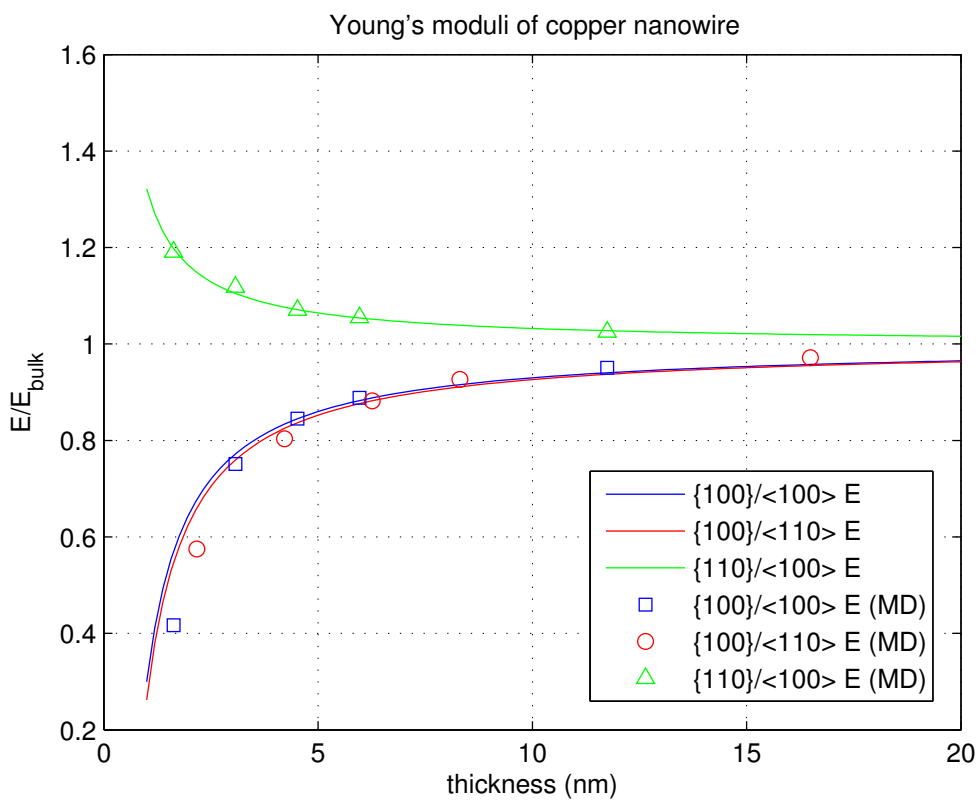

Figure 7. Size-dependent Young's moduli of $<100>/\{100\},<100>/\{110\},<110>/\{100\}$ copper nanowires.

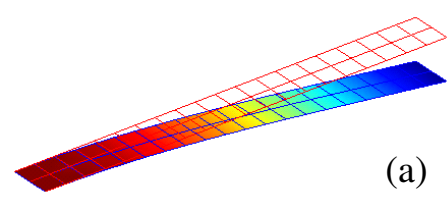

(a)

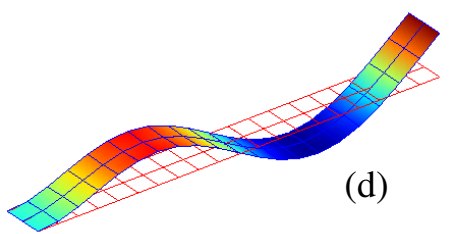

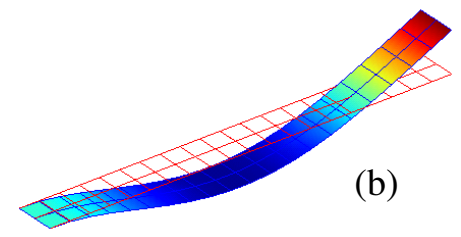

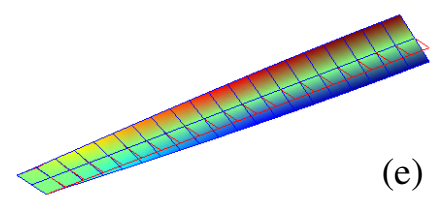

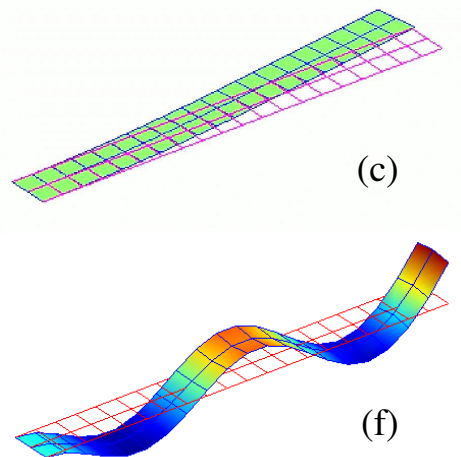

(f)

Figure 8. Mesh configuration and vibration modes of a clamped beam: (a) first mode,

(b) second mode, (c) third mode, (d) fourth mode, (e) fifth mode without surface effect,

(f) fifth mode with surface effect.

integration scheme is applied in order to avoid numerical transverse shear locking. Four-point Gaussian integration is used for the membrane and bending component, and two-point Gaussian integration is used for the transverse shear component: $2 \times 1$ for $\gamma_{12}$ and $1 \times 2$ for $\gamma_{13}$. The shear correction factor $k$ in (34) is set to $\frac{5}{6}$ in the finite element analysis. The natural frequencies of clamped beams, obtained through finite element analysis, are listed in Table 4.

The natural frequencies with surface effect are smaller than those without surface effect in all test cases. The surface effect increases as the thickness decreases. When the thickness is $3 \mathrm{~nm}$, the natural 


\begin{tabular}{|cccc|}
\hline $\begin{array}{c}\text { Thickness } \\
(\mathrm{nm})\end{array}$ & \multicolumn{3}{c|}{ Natural frequency $(\mathrm{MHz})$} \\
3 & 14.4 & 10.0 & $69.4 \%$ \\
4.5 & 21.6 & 18.4 & $84.9 \%$ \\
6 & 28.8 & 26.0 & $90.1 \%$ \\
12 & 57.4 & 55.0 & $95.9 \%$ \\
\hline
\end{tabular}

Table 4. Natural frequencies of clamped beams without and with surface effect. The length and the width of the beams are $300 \mathrm{~nm}$ and $30 \mathrm{~nm}$, respectively.

\begin{tabular}{|ccccc|}
\hline Thickness & Length & \multicolumn{3}{c|}{ Natural frequency $(\mathrm{GHz})$} \\
$(\mathrm{nm})$ & $(\mathrm{nm})$ & w/o surf. & w/ surf. & MD sim. \\
1.6 & 11.7 & $4.95(4.99)$ & $1.85(1.86)$ & 2.55 \\
3.1 & 23.3 & $2.37(2.39)$ & $1.75(1.77)$ & 1.77 \\
4.5 & 34.9 & $1.55(1.57)$ & $1.29(1.31)$ & 1.26 \\
6.0 & 46.5 & $1.15(1.17)$ & $1.00(1.02)$ & 1.01 \\
11.7 & 92.7 & $0.56(0.59)$ & $0.52(0.54)$ & 0.50 \\
\hline
\end{tabular}

Table 5. Natural frequencies of a $\{100\} /<100>$ nanowire with one clamped edge. The width is equal to the thickness, and the length is about eight times the thickness. Numbers in parentheses indicate the analytic solution calculated using (81).

frequency with surface effect is $69 \%$ of the natural frequency without surface effect. Figure 8 shows the vibration modes of a clamped beam with $3 \mathrm{~nm}$ thickness. From first to fourth vibration modes, test cases without and with surface effects yield the same results, as shown in Figure 8(a) to 8(d). However, the fifth vibration mode with surface effect (see Figure 8(f)) is different from the fifth vibration mode without surface effect (see Figure 8(e)).

Another example of beam vibration is solved using beam finite element analysis. Two-node beam elements are used, and a selective reduced integral scheme is applied to avoid shear locking. Two-point Gaussian integration is used for the bending component and one-point Gaussian integration for transverse shear component. The shear correction factor $k$ in (74) is set to $\frac{5}{6}$ in the finite element analysis. The natural frequencies of $<100>/\{100\}$ nanowires with different thicknesses are obtained via FEM and MD simulation and listed in Table 5. The width of each nanowire is fixed to be equal to the thickness, and the length is eight times the thickness.

The natural frequencies of the clamped beams can be calculated analytically by the following equation [Weaver et al. 1990]:

$$
f=\frac{1}{2 \pi}\left(\frac{1.875}{L}\right)^{2} \sqrt{\frac{E I}{\rho A}},
$$

where $L$ is the length, $A$ is the cross-sectional area, $\rho$ is the density, $E$ is the Young's modulus, and $I$ is the second moment of area. In order to consider surface effect, the effective bending rigidity of a nanowire in (76) is used in the analysis. 
The FEM results and analytical solutions show good agreement in all cases, both with and without surface effect. The results with surface effect also agree very well with MD results, except when the thickness is $1.6 \mathrm{~nm}$. The main cause of this exception is that the estimated effective bending rigidity drops rapidly when the thickness of a nanowire reaches $1.6 \mathrm{~nm}$.

\section{Equilibrium strain and surface stress}

The lattice spacing of a relaxed nanofilm is different from that of bulk material, due to the surface stress on the top and bottom surfaces. A similar phenomenon can occur with nanowires. The equilibrium strain in the relaxed state can be calculated using initial surface stress and surface elastic tensor. In addition, if the equilibrium strain of a nanofilm is known, the surface stress can be calculated from the equilibrium strain through a matching technique. In this section, surface stress is calculated using equilibrium strain as measured by the MD simulation.

4.1. Surface stress of copper nanofilms. Assume that the surface types of top and bottom surfaces are the same and that there is no external force on the film. The relationship between equilibrium strain $\epsilon^{*}$ and surface stress can be derived from (34), as follows:

$$
\begin{gathered}
\delta U=\delta \boldsymbol{d}^{T} \boldsymbol{B}_{m}^{T}\left(\left[\boldsymbol{A}^{\prime}\right] \boldsymbol{B}_{m} \boldsymbol{d}+2\left\{\tau^{0}\right\}\right)=0, \\
{\left[\boldsymbol{A}^{\prime}\right]\left\{\begin{array}{c}
\epsilon_{11}^{*} \\
\epsilon_{22}^{*} \\
2 \epsilon_{12}^{*}
\end{array}\right\}+2\left\{\begin{array}{l}
\tau_{11}^{0} \\
\tau_{22}^{0} \\
\tau_{12}^{0}
\end{array}\right\}=0,}
\end{gathered}
$$

where $\left[\boldsymbol{A}^{\prime}\right]=h[\boldsymbol{Q}]+2[\boldsymbol{T}]$.

To obtain the equilibrium strain of a nanofilm, an MD simulation was performed using an NPT ensemble at a temperature of $0.1 \mathrm{~K}$. The thickness of the MD model was set to $3.1 \mathrm{~nm}$ for the $\{100\} /<100>$ and $\{100\} /<110>$ nanofilms and $5.2 \mathrm{~nm}$ for the $\{111\} /<110>$ nanofilm. The $\{110\} /<100>$ nanofilm was $4.2 \mathrm{~nm}$ thick. The initial surface stress in Table 6 was calculated by applying the one-point matching method to the equilibrium strain obtained via an MD simulation. The shear component, $\tau_{12}^{0}$, which is not shown in Table 6, is zero for all the tested surfaces.

The equilibrium strain of a copper nanofilm as a function of thickness is shown in Figure 9 and 10. The lines represent the results of the proposed continuum-based model, and the marks show the results of an MD simulation. The results for $\{100\} /<100>,\{100\} /<110>$, and $\{111\} /<110>$ nanofilms are illustrated in Figure 9. Lines e1 and e2, denoted in Figure 10, are the equilibrium strains in the $<100>$ and $<110>$ directions, respectively, of the $\{110\} /<100>$ nanofilm.

\begin{tabular}{|ccccc|}
\hline & $\{100\} /<100>$ & $\{100\} /<110>$ & $\{111\} /<110>$ & $\{110\} /<100>$ \\
$\tau_{11}^{0}$ & 1.5034 & 1.3806 & 0.8717 & 1.1149 \\
$\tau_{22}^{0}$ & 1.5034 & 1.3806 & 0.8717 & 0.9993 \\
\hline
\end{tabular}

Table 6. Initial surface stress of nanofilms, calculated by the one-point matching method (in $\mathrm{N} / \mathrm{m}$ ). 


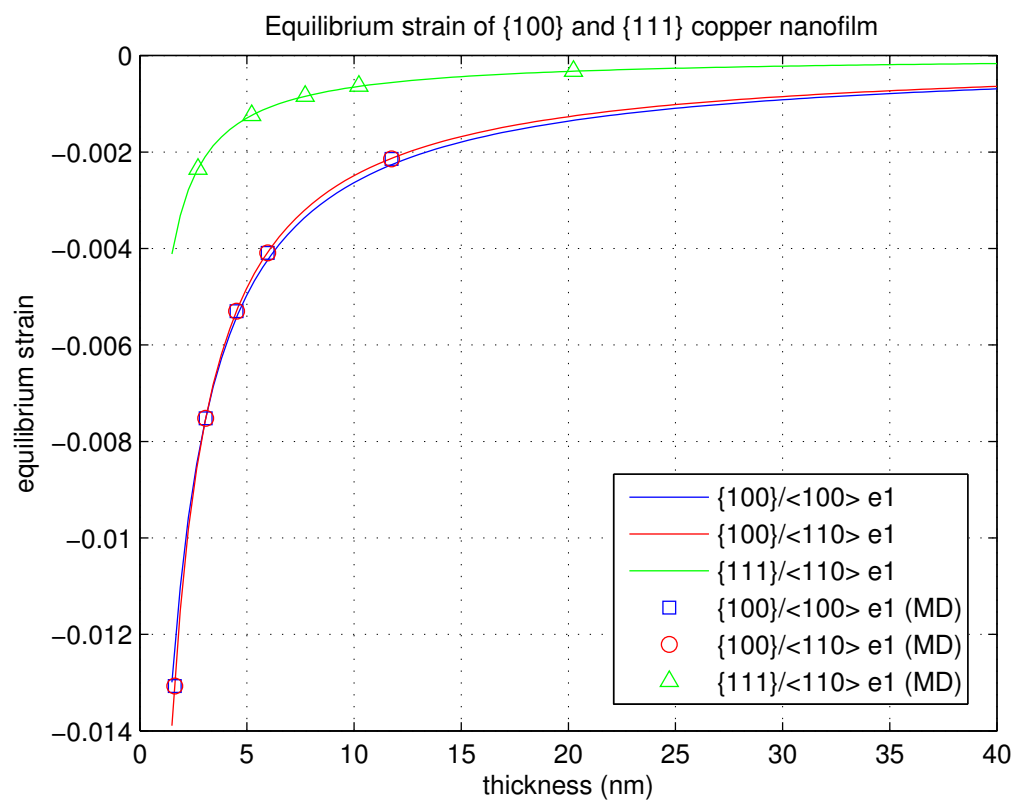

Figure 9. Equilibrium strain of $\{100\} /<100>,\{100\} /<110>$, and $\{111\} /<110>$ copper nanofilms.

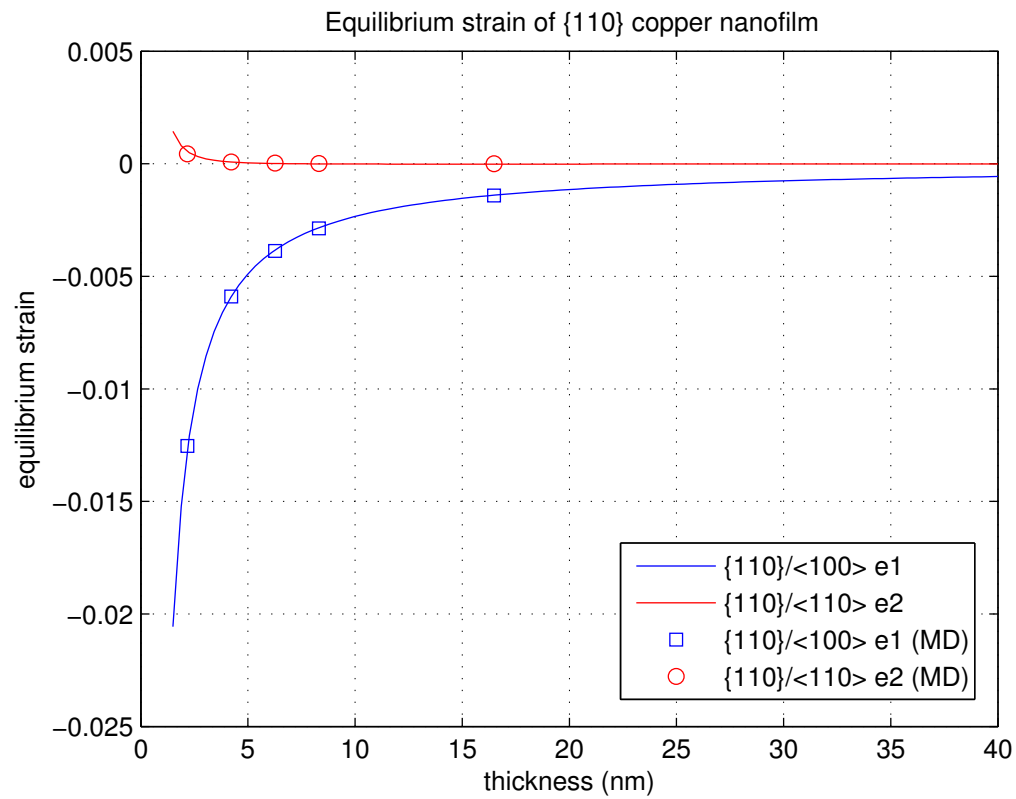

Figure 10. Equilibrium strain of a $\{110\} /<100>$ copper nanofilm.

4.2. Surface stress of copper nanowires. The initial surface stress of a nanowire can be calculated using equilibrium strain in a manner analogous to the case of a nanofilm. The relationship between equilibrium strain and surface stress is given as follows: 


\begin{tabular}{|cccc|}
\hline & $<100>/\{100\}$ & $<100>/\{110\}$ & $<110>/\{100\}$ \\
$\tau_{x x}^{y 0}$ & 0.6984 & 0.7047 & 1.5670 \\
$\tau_{x x}^{z 0}$ & 0.6984 & 0.7047 & -0.0178 \\
\hline
\end{tabular}

Table 7. Initial surface stress of nanowires calculated by a matching method (in $\mathrm{N} / \mathrm{m}$ ).

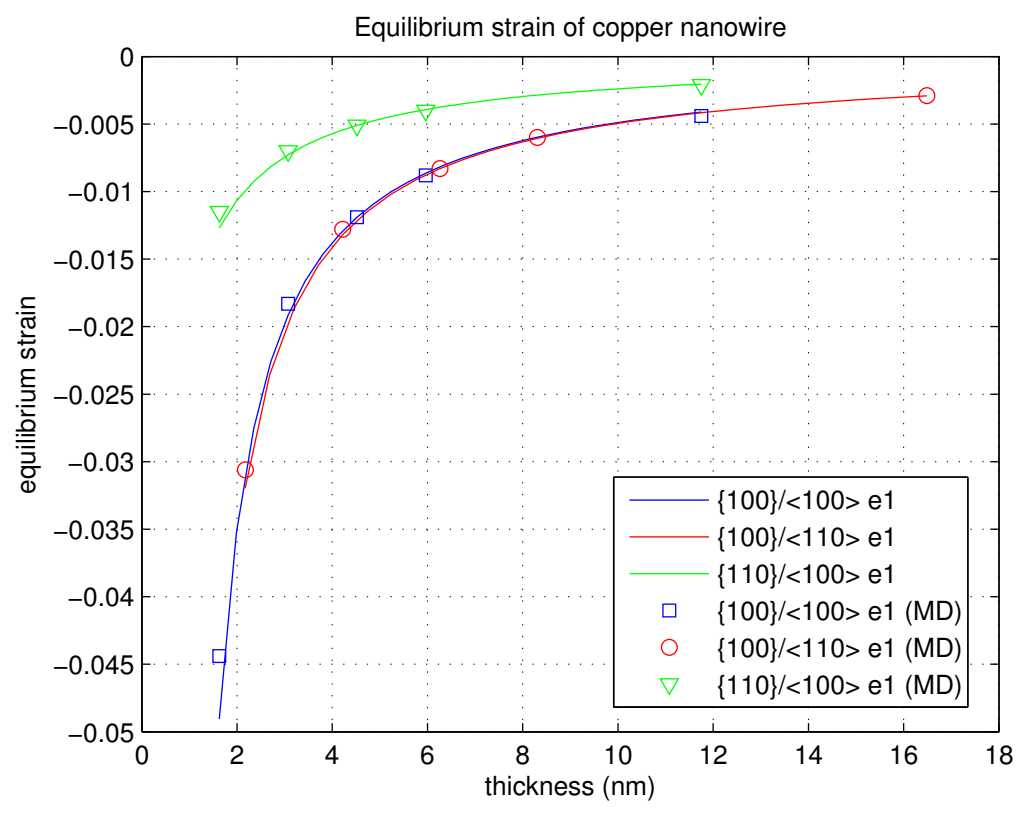

Figure 11. Equilibrium strain of $<100>/\{100\},<100>/\{110\}$, and $<110>/\{100\}$ copper nanowires.

$$
\left[b h\left(E_{1}+\frac{2}{b} T_{1111}^{y}+\frac{2}{h} T_{1111}^{z}\right)\right]\left\{\epsilon_{x}^{*}\right\}+\left\{2 h \tau_{x x}^{y 0}+2 b \tau_{x x}^{z 0}\right\}=0,
$$

where $\epsilon_{x}^{*}$ is the equilibrium strain of a nanowire, $\tau_{x x}^{y 0}$ and $\tau_{x x}^{z 0}$ are initial surface stress components of the $y$ - and $z$-surfaces, and $b$ and $h$ are the width and thickness of a nanowire, respectively.

The equilibrium strains present in $<100>/\{100\},<100>/\{110\}$, and $<110>/\{100\}$ nanowires were obtained by an MD simulation. The cross-sectional dimensions of the MD models are $b=h=4.5 \mathrm{~nm}$ for the $\langle 100>/\{100\}$ nanowire and $b=h=6.3 \mathrm{~nm}$ for the $<100>/\{110\}$ nanowire. For the $<110>/\{100\}$ nanowire, an MD simulation was carried out twice with two different widths, $b=4.5 \mathrm{~nm}$ and $b=8.9 \mathrm{~nm}$, and a constant thickness of $h=6.3 \mathrm{~nm}$. The initial surface stress, calculated from the equilibrium strain, is listed in Table 7.

The equilibrium strain of a nanowire can be calculated from the initial surface stress using (84). These results are shown in Figure 11. The calculated equilibrium strains represented by lines in the figure have good agreement with the mark data obtained by MD simulation.

\section{Conclusion}

Multiscale continuum models and corresponding finite elements were developed for both nanofilms and nanowires subject to an anisotropic surface effect. In order to consider the anisotropic surface effect, 
linear surface elasticity was used instead of the isotropic surface elasticity proposed by Gurtin and Murdoch. This linear surface elasticity was represented by initial surface stress and surface elastic tensor.

The surface elastic tensor of a nanofilm was calculated via the one-point matching method and an MD simulation. The initial surface stress was estimated using the equilibrium strain, which in turn was computed via MD simulation. Four different orthotropic nanofilms were considered as numerical examples, with $\{100\} /<100>,\{100\} /<110>,\{111\} /<110>$, and $\{110\} /<100>$ surfaces. The size-dependent elastic modulus and equilibrium strain values calculated by the proposed continuum model were in good agreement with the results of the MD simulation. However, there were slight discrepancies between these surface parameters (initial surface stress and surface elastic tensor) as calculated by the matching technique and their corresponding reference values [Shenoy 2005; Dingreville and Qu 2007]. This is because the presented surface parameters include the hyperelastic effect of the bulk material as well as the surface effect itself.

For a nanowire, surface parameters were estimated using either the one-point or two-point matching method. $<100>/\{100\},<100>/\{110\}$, and $<110>/\{100\}$ nanowires with rectangular cross-sections were considered as numerical examples. In the calculation of surface parameters, only one MD simulation was used for the $\langle 100>/\{100\}$ and $<100>/\{110\}$ nanowires, whereas two MD simulations were performed for the $\langle 110>/\{100\}$ nanowire because it had different surfaces in the $y$-and $z$-directions. The results from the continuum-based model and the MD simulation correlated very well.

In this paper, only rectangular cross-sectional nanowires were considered. If the shape of a crosssection is not rectangular, the surface component of the internal virtual work will change. Since a nanowire with a circular cross-section presents a special case, it might not always be feasible to apply the approach described in this paper. However, in most cases, this approach will work because a crystalline nanowire tends to have a multifaceted cross-section rather than a circular shape. Moreover, a nanowire could undergo surface reconstruction and could have various surfaces which are not low-index surfaces. If a nanowire has a surface different from those mentioned in this paper, initial surface stress and surface stress tensor will change. However, initial surface stress and surface stress tensor can still be evaluated using the MD-based matching method proposed in this paper. Therefore, the proposed model can be applied to nanowires with various kinds of surfaces.

The proposed multiscale continuum model could prove useful in the development of a finite element method including surface effect, and it is expected to be a good design tool for nanoscale structures in which the surface effect is dominant.

\section{Acknowledgments}

This research was supported by the World Class University (WCU) program (R31-2008-000-10083-0) and a National Research Foundation of Korea grant (No. 2012R1A3A2048841) funded by the Korea government (MEST).

\section{References}

[Cammarata and Sieradzki 1989] R. C. Cammarata and K. Sieradzki, "Effects of surface stress on the elastic moduli of thin films and superlattices", Phys. Rev. Lett. 62 (1989), 2005-2008.

[Cho et al. 2009] M. Cho, J. Choi, and W. Kim, "Continuum-based bridging model of nanoscale thin film considering surface effects”, Jap. J. Appl. Phys. 48 (2009), 020219. 
[Choi et al. 2010a] J. Choi, M. Cho, and W. Kim, "Multiscale analysis of nano-scale thin film considering surface effects: thermomechanical properties", J. Mech. Mat. Struct. 5 (2010), 161-183.

[Choi et al. 2010b] J. Choi, M. Cho, and W. Kim, "Surface effects on the dynamic behavior of nano-sized thin film resonator", Appl. Phys. Lett. 97 (2010), 171901.

[Daw and Baskes 1984] M. S. Daw and M. I. Baskes, "Embedded-atom method: derivation and application to impurities, surfaces, and other defects in metals", Phys. Rev. B 29 (1984), 6443-6453.

[Diao et al. 2004] J. Diao, K. Gall, and M. L. Dunn, "Atomistic simulation of the structure and elastic properties of gold nanowires", J. Mech. Phys. Solids 52 (2004), 1935-2186.

[Dingreville and Qu 2007] R. Dingreville and J. Qu, "A semi-analytical method to compute surface elastic properties", Acta Mater. 55 (2007), 141-147.

[Dingreville et al. 2005] R. Dingreville, J. Qu, and M. Cherkaoui, "Surface free energy and its effect on the elastic behavior of nano-sized particles, wires and films", J. Mech. Phys. Solids 53 (2005), 1827-1854.

[Dingreville et al. 2008] R. Dingreville, A. J. Kulkarni, M. Zhou, and J. Qu, "A semi-analytical method for quantifying the size-dependent elasticity of nanostructures", Modelling Simul. Mater. Sci. Eng. 16 (2008), 025002.

[Foiles et al. 1986] S. M. Foiles, M. I. Baskes, and M. S. Daw, "Embedded-atom method functions for the fcc metals Cu, Ag, Au, Ni, Pd, Pt, and their alloys", Phys. Rev. B 33 (1986), 7983-7991.

[Guo and Zhao 2005] J.-G. Guo and Y.-P. Zhao, "The size-dependent elastic properties of nanofilms with surface effects”, $J$. Appl. Phys. 98 (2005), 074306.

[Gurtin and Murdoch 1975] M. E. Gurtin and A. I. Murdoch, "A continuum theory of elastic material surfaces", Arch. Rat. Mech. Anal. 57 (1975), 291-323.

[Gurtin and Murdoch 1978] M. E. Gurtin and A. I. Murdoch, "Surface stress in solids", Int. J. Solid Struct. 14 (1978), 431-440.

[He and Lilley 2008] J. He and C. M. Lilley, "Surface stress effect on bending resonance of nanowires with different boundary conditions", Appl. Phys. Lett. 93 (2008), 263108.

[Kim and Cho 2010] W. Kim and M. Cho, "Surface effect on the self-equilibrium state and size-dependent elasticity of FCC thin films", Modelling Simul. Mater. Sci. Eng. 18 (2010), 085006.

[Liang et al. 2005] H. Liang, M. Upmanyu, and H. Huang, "Size-dependent elasticity of nanowires: nonlinear effects", Phys. Rev. B 71 (2005), 241403.

[Lim and He 2004] C. W. Lim and L. H. He, "Size-dependent nonlinear response of thin elastic films with nano-scale thickness", Int. J. Mech. Sci. 46 (2004), 1715-1726.

[Lu et al. 2006] P. Lu, L. H. He, H. P. Lee, and C. Lu, “Thin plate theory including surface effects", Int. J. Solid Struct. 43 (2006), 4631-4647.

[Miller and Shenoy 2000] R. E. Miller and V. B. Shenoy, "Size-dependent elastic properties of nanosized structural elements", Nanotechnology 11 (2000), 139-147.

[Park 2009] H. S. Park, "Quantifying the size-dependent effect of the residual surface stress on the resonant frequencies of silicon nanowires if finite deformation kinematics are considered", Nanotechnology 20 (2009), 115701.

[Plimpton 1995] S. J. Plimpton, "Fast parallel algorithms for short-range molecular dynamics", J. Comput. Phys. 117 (1995), $1-19$.

[Rapaport 2004] D. C. Rapaport, The art of molecular dynamics simulation, pp. 15-17, Cambridge University Press, 2004.

[Shenoy 2005] V. B. Shenoy, "Atomistic calculations of elastic properties of metallic fcc crystal surfaces", Phys. Rev. B 71 (2005), 094104.

[Streitz et al. 1994] F. H. Streitz, R. C. Cammarata, and K. Sieradzki, "Surface-stress effects on elastic properties, I: thin metal films", Phys. Rev. B 49 (1994), 10699-10706.

[Subramaniyan and Sun 2008] A. K. Subramaniyan and C. T. Sun, "Continuum interpretation of virial stress in molecular simulations", Int. J. Solids Struct. 45 (2008), 4340-4346.

[Sun and Zhang 2005] C. T. Sun and H. Zhang, "Size-dependent elastic moduli of platelike nanomaterials", J. Appl. Phys. 93 (2005), 1212-1218. 
[Wang et al. 2008] J. Wang, Q.-A. Huang, and H. Yu, "Size and temperature dependence of Young's modulus of a silicon nano-plate", J. Phys. D: Appl. Phys. 41 (2008), 165406.

[Weaver et al. 1990] W. Weaver, Jr., S. P. Timoshenko, and D. H. Young, Vibration problems in engineering, 5th ed., pp. 416-432, John Wiley \& Sons, 1990.

[Wolf 1991] D. Wolf, "Surface-stress-induced structure and elastic behavior of thin films", Appl. Phys. Lett. 58 (1991), 20812083.

[Zhang et al. 2008] T.-Y. Zhang, M. Luo, and W. K. Chan, "Size-dependent surface stress, surface stiffness, and Young's modulus of hexagonal prism [111] beta-SiC nanowires", J. Appl. Phys. 103 (2008), 104308.

[Zhou and Huang 2004] L. G. Zhou and H. Huang, "Are surfaces elastically softer or stiffer?", Appl. Phys. Lett. 84 (2004), 1940-1942.

Received 9 Jun 2011. Revised 1 Jun 2012. Accepted 18 Jun 2012.

WONBAE KiM: wbkim@snu.ac.kr

Division of WCU Multiscale Mechanical Design, School of Mechanical and Aerospace Engineering, Seoul National University, Seoul 151-744, South Korea

SEUNG YUn RHEE: syrhee8@snu.ac.kr

Aircraft Certification Team, Aerospace Safety and Certification Center, Korea Aerospace Research Institute, 169-84 Gwahangno, Yuseong-gu, Daejeon 305-806, South Korea

MAENGHYO CHO: mhcho@snu.ac.kr

Division of WCU Multiscale Mechanical Design, School of Mechanical and Aerospace Engineering, Seoul National University, Seoul 151-744, South Korea

http://ssnd.snu.ac.kr/members/professor.html 


\title{
JOURNAL OF MECHANICS OF MATERIALS AND STRUCTURES
}

\author{
jomms.net
}

\author{
Founded by Charles R. Steele and Marie-Louise Steele \\ EDITORS \\ Charles R. STEele \\ DAVIDE BIGONI \\ Stanford University, USA \\ University of Trento, Italy \\ IWONA JASIUK University of Illinois at Urbana-Champaign, USA \\ YASUHIDE SHINDO Tohoku University, Japan
}

\section{EDITORIAL BOARD}

H. D. Bui École Polytechnique, France

J. P. CARTER University of Sydney, Australia

R. M. CHRISTENSEN Stanford University, USA

G. M. L. GLadWELL University of Waterloo, Canada

D. H. Hodges Georgia Institute of Technology, USA

J. Hutchinson Harvard University, USA

C. HwU National Cheng Kung University, Taiwan

B. L. KARIHALOO University of Wales, UK

Y. Y. KIM Seoul National University, Republic of Korea

Z. Mroz Academy of Science, Poland

D. PAmplona Universidade Católica do Rio de Janeiro, Brazil

M. B. RUBIN Technion, Haifa, Israel

A. N. SHUPIKOV Ukrainian Academy of Sciences, Ukraine

T. TARNAI University Budapest, Hungary

F. Y. M. WAN University of California, Irvine, USA

P. WRIGGERS Universität Hannover, Germany

W. YANG Tsinghua University, China

F. ZIEGLER Technische Universität Wien, Austria

PRODUCTION production@msp.org

SILVIO LEVY Scientific Editor

See http://jomms.net for submission guidelines.

JoMMS (ISSN 1559-3959) is published in 10 issues a year. The subscription price for 2012 is US $\$ 555 /$ year for the electronic version, and \$735/year (+\$60 shipping outside the US) for print and electronic. Subscriptions, requests for back issues, and changes of address should be sent to Mathematical Sciences Publishers, Department of Mathematics, University of California, Berkeley, CA 94720-3840.

JoMMS peer-review and production is managed by EditFLow ${ }^{\circledR}$ from Mathematical Sciences Publishers.

\author{
mathematical sciences publishers \\ http://msp.org/ \\ A NON-PROFIT CORPORATION \\ Typeset in $\mathrm{IAT}_{\mathrm{E}} \mathrm{X}$ \\ Copyright $@ 2012$ by Mathematical Sciences Publishers
}




\section{Journal of Mechanics of Materials and Structures}

\section{Volume 7, No. $7 \quad$ September 2012}

Molecular dynamics-based continuum models for the linear elasticity of nanofilms and nanowires with anisotropic surface effects

Wonbae Kim, Seung Yun Rhee and Máenghyo Cho

Characterization of human skin through skin expansion

Djenane C. Pamplona and Claudio R. Carvalho 641

In-plane dynamic excitation of AAC masonry walls patched with FRP: dynamic testing and analysis

DVIR ELMALICH and ODED RABINOVITCH

Hygrothermal analysis of exponentially graded rectangular plates

ASHRAF M. ZENKOUR 687

Green's function for symmetric loading of an elastic sphere with application to contact problems ALEXeY S. TITOVICH and ANDREW N. NORRIS 701

Multi-hit armour characterisation of metal-composite bi-layers

K. Karthikeyan, B. P. Russell, V. S. Deshpande and N. A. Fleck 